\title{
The Corolla Polynomial for spontaneously Broken Gauge Theories
}

\author{
David Prinz*, \\ Institut für Mathematik und Institut für Physik, \\ Humboldt-Universität zu Berlin
}

August 8, 2018

\begin{abstract}
In 1, 2, 3] the Corolla Polynomial $\mathcal{C}(\Gamma) \in \mathbb{C}\left[a_{h_{1}}, \ldots, a_{h_{\mid \Gamma^{[1 / 2]}}}\right]$ was introduced as a graph polynomial in half-edge variables $\left\{a_{h}\right\}_{h \in \Gamma^{[1 / 2]}}$ over a 3-regular scalar quantum field theory (QFT) Feynman graph $\Gamma$. It allows for a covariant quantization of pure Yang-Mills theory without the need for introducing ghost fields, clarifies the relation between quantum gauge theory and scalar QFT with cubic interaction and translates back the problem of renormalizing quantum gauge theory to the problem of renormalizing scalar QFT with cubic interaction (which is super renormalizable in 4 dimensions of spacetime). Furthermore, it is, as we believe, useful for computer calculations. In 4 on which this paper is based 1 the formulation of 1, 2, 3, gets slightly altered in a fashion specialized in the case of the Feynman gauge. It is then formulated as a graph polynomial $\mathcal{C}(\Gamma) \in \mathbb{C}\left[a_{h_{1 \pm}}, \ldots, a_{h_{\left|\Gamma^{[1 / 2]}\right|^{ \pm}}}, b_{h_{1}}, \ldots, b_{h_{\left|\Gamma^{[1 / 2]}\right|}}\right]$ in three different types of half-edge variables $\left\{a_{h_{+}}, a_{h_{-}}, b_{h}\right\}_{h \in \Gamma^{[1 / 2]}}$. This formulation is also suitable for the generalization to the case of spontaneously broken gauge theories (in particular all bosons from the Standard Model), as was first worked out in [4 and gets reviewed here.
\end{abstract}

\section{Introduction}

\subsection{Motivation}

One of the curiosities of non-abelian gauge theories is the observation that gauge bosons in the quantized theory do not only possess the two experimentally verified transversal degrees of freedom. In addition, there is also a longitudinal one. In quantum electrodynamics photons also possess a longitudinal degree of freedom. The Ward-Takahashi identity then ensures that this longitudinal degree of freedom is cancelled with the corresponding timelike degree of freedom. Therefore, the complete photon amplitude is purely transversal. In non-abelian gauge theories this problem was solved by the introduction of unphysical particles? 2 , existing only in closed loops, the so-called ghosts. Then, the Slavnov-Taylor identity ensures that all gauge boson amplitudes are purely transversal. Although it was shown that this could be done in a self-consistent way, it remained unsatisfying since there was no convenient argument despite adjusting the theory to experimental facts. However, this question is solved in the definition of the Corolla polynomial 2 by the introduction of cycle homology and allows, as such, a covariant quantization of gauge fields without the need of introducing ghost fields [2, 5, 6, 7].

\footnotetext{
*prinz@physik.hu-berlin.de

${ }^{1}$ For reasons of clarity and comprehensibility [4] will not be given explicitly as a reference every time below, even if most passages are literally quoted.

${ }^{2}$ In the sense of contradicting the Spin-Statistic theorem, i.e. being scalar particles (having spin 0) but obeying Fermi-Dirac-statistics [5, 6].
} 
Furthermore, the introduction of the Corolla polynomial also clarifies the relation between scalar QFT with cubic interaction and quantum gauge theory. This is done using the parametric representation with its two Kirchhoff or Symanzik polynomials and the creation of a Corolla differential out of the Corolla polynomial making implicit use of graph homology [2. In particular, the parametric integrand for all gauge theory Feynman graphs, which can be created from a 3-regular scalar QFT Feynman graph $\Gamma$ by shrinking edges, can be obtained whilst acting with the Corolla differential $\mathcal{D}_{\mathrm{QCD}}(\Gamma)$ on the parametric integrand $I(\Gamma)$ such that the parametric integrand for gauge theory $\tilde{I}_{\mathcal{F}}(\Gamma)[2,3]$ read@ 3$]$

$$
\tilde{I}_{\mathcal{F}}(\Gamma)=\mathcal{D}_{\mathrm{QCD}}(\Gamma) I(\Gamma) .
$$

It is then possible to receive the renormalized quantum gauge theory amplitude by replacing $I(\Gamma)$ by its renormalized analogue $I^{R}(\Gamma)$, i.e. the problem of renormalizing gauge theory gets translated back to the renormalization of scalar QFT with cubic interaction (which is super renormalizable in 4 dimensions of spacetime). This is a simple consequence of Leibniz's rule for differentiation under the integral sign [2, 5].

The aim of this paper is now to review the generalization worked out in [4] to the approach of [1, 2, 3] to include the gauge bosons of the electroweak sector of the Standard Model (cf. Subsection 5.2) as well as its scalar particles (cf. Subsection 5.3). This was achieved by first working out the combinatorics of labeling a 3-regular scalar QFT Feynman graph with labels of the gauge bosons of the electroweak sector of the Standard Model and then by working out the additional tensor structures arising from the inclusion of the Feynman rules for the scalar particles of the electroweak sector of the Standard Model [4, 8].

\subsection{Organization of this Paper}

This paper is organized as follows: First we set up the notations and conventions in Section 2 , Then, we introduce the necessary graph theoretic notions in Section 3, thereby allowing us to define the parametric representation in a slightly different way which suits our purposes in Section 4. With this knowledge, we are then able to introduce the several Corolla Polynomials and Differentials in Section 5. We then end, in Section 6, with a short conclusion.

\section{Notations and Conventions}

\subsection{Einstein summation Convention}

Important in this paper and also in [2, 3, 4] is that Einstein summation convention is even assumed (if not indicated otherwise) whenever two Lorentz indices are the same - independent of if they correspond to the space of covariant or contravariant vectors:

$$
\eta^{\mu \nu} \eta^{\nu \rho}:=\eta^{\mu \nu_{1}} \eta_{\nu_{1} \nu_{2}} \eta^{\nu_{2} \rho}=\eta^{\mu \rho}
$$

This at first sight inconvenient looking expression allows to define the Corolla polynomial in a more elegant way, cf. Remark 5.2.

\subsection{Feynman Rules, chosen Gauge and Dimension of Spacetime}

We use the definitions for the Feynman rules given in [8] with all appearing signs chosen positive, i.e. $\eta_{G}=\eta_{s}=\eta_{e}=\eta=\eta_{Z}=1$. Furthermore, we use the Feynman gauge throughout this paper - this allows a more compact notation and avoids unnecessary applications of the Leibniz rule,

\footnotetext{
${ }^{3}$ Actually the different quantum gauge theory graph contributions created by the Corolla differential are hidden in the parametric integrand $\tilde{I}_{\mathcal{F}}(\Gamma)$ in its regular part and residues along the Schwinger parameters, cf. Subsection 5.1 (mainly Theorem [5.7).
} 
i.e. $\xi_{G}=\xi_{A}=\xi_{W}=\xi_{Z}=1$, cf. Remark 5.2. The relevant Feynman rules with this conventions are explicitly listed in [4, Appendix A]. Furthermore, we work in a 4-dimensional spacetime for concreteness, even though the Corolla polynomial is not limited to that choice.

\subsection{Feynman Graphs with oriented Edges}

In the Standard Model some particle types have oriented edges (fermions, ghosts, $W^{ \pm}$-particles, $\varphi^{ \pm}$-particles). We work in this paper with unoriented edges, where a graph with unoriented edges is understood as the sum of all graphs with all possible edge orientations times their corresponding symmetry factor (cf. Definition 3.3).

\subsection{Coupling Constants}

In order to avoid confusion between Euler's number and the electric charge, we underline all coupling constants.

\section{Graph theoretic Notions}

A QFT is characterized by its Lagrangian density which dictates the sets $\mathcal{R}_{V}$ and $\mathcal{R}_{E}$ of all possible vertex- and edge-types, respectively [5]. This states all allowed particle interactions and particle types out of which Feynman graphs can be built of. Now, we provide all necessary graph theoretic notions:

Definition 3.1 (Feynman graph, in parts literally quoted from [9, Definition 1]). A Feynman graph $\Gamma$ is characterized by a set of vertices $\Gamma^{[0]}$ and a set of edges $\Gamma^{[1]}=\Gamma_{\text {ext }}^{[1]} \cup \Gamma_{\text {int }}^{[1]}$ whose elements are part of $\mathcal{R}_{V}$ and $\mathcal{R}_{E}$, respectively, and a set of maps

$$
\partial_{j}: \quad \Gamma^{[1]} \mapsto \Gamma^{[0]} \cup\{1,2, \ldots, N\}, \quad j \in\{0,1\},
$$

respecting the vertex and edge types given by $\mathcal{R}_{V}$ and $\mathcal{R}_{E}$. Furthermore, the case $\partial_{0}$ and $\partial_{1}$ being both in $\{1,2, \ldots, N\}$ is excluded. The set $\{1,2, \ldots, N\}$ labels external edges of $\Gamma$, such that $\sum_{j=0}^{1} \operatorname{card} \partial_{j}^{-1}(v)=1$ for all $v \in\{1,2, \ldots, N\}$. The set of external edges is therefore defined as $\Gamma_{\text {ext }}^{[1]}:=\bigcup_{j=0}^{1} \partial_{j}\{1,2, \ldots, N\}$ and the set of internal edges as its complement with respect to the set of edges of $\Gamma$, i.e. $\Gamma_{\text {int }}^{[1]}:=\Gamma^{[1]} \backslash \Gamma_{\text {ext }}^{[1]}$. Therefore, external edges can be labeled by $e_{1}, e_{2}, \ldots, e_{N} \in \Gamma_{\text {ext }}^{[1]}$, with $e_{k}:=\bigcup_{j=0}^{1} \partial_{j}(k)$.

Furthermore, we omit scalar graphs with edges which form self-loops (so-called tadpoles). This is possible without loss of generality since their amplitudes vanish during the renormalization process 4 . In the gauge theory amplitudes created by the Corolla polynomial, amplitudes from gauge theory graphs with tadpoles will show up again by the use of graph-homology, cf. Section 5 and [2].

The two Symanzik polynomials (Definition 4.3 and Definition 4.5) are polynomials in edgevariables $\left\{A_{e}\right\}_{e \in \Gamma^{[1]}}$, whereas the Corolla polynomial (Definition [5.1) is a polynomial in halfedge-variables $\left\{a_{h_{+}}, a_{h_{-}}, b_{h}\right\}_{h \in \Gamma^{[1 / 2]}}$ which are defined as follows:

\footnotetext{
${ }^{4}$ Speaking in Hopf-algebraic language, the graphs with tadpoles create an Hopf ideal $I_{\text {tad }}$ in the Hopf-algebra $H_{\mathrm{FG}}$ of Feynman graphs and we can effectively work in the quotient space $H_{\mathrm{FG}} / I_{\mathrm{tad}}[2]$.
} 
Definition 3.2 (Half-edge [2]). Let $\Gamma$ be a Feynman graph, $\Gamma^{[0]}$ the set of its vertices, $\Gamma^{[1]}$ the set of its edges and $n(v) \subset \Gamma^{[1]}$ the set of edges adjacent to the vertex $v$. Then the tuple

$$
h:=(v, e), \quad v \in \Gamma^{[0]}, e \in n(v),
$$

is called a half-edge of $\Gamma$. The set of all half-edges of $\Gamma$ is denoted by $\Gamma^{[1 / 2]}$. Note that each internal edge defines two half-edges in a unique way, since we do not allow tadpoles (cf. Definition 3.1.).

Next, we define automorphisms of a Feynman graph $\Gamma$ and its symmetry factor $\operatorname{sym}(\Gamma)$ :

Definition 3.3 (Automorphisms and symmetry factors of a Feynman graph, in parts literally quoted from [9, Definition 2]). Let $\Gamma$ be a Feynman graph. An automorphism $g$ of $\Gamma$ is given by an automorphism $g^{[0]}$ of $\Gamma^{[0]}$ and an automorphism $g^{[1]}$ of $\Gamma^{[1]}$ that is the identity on $\Gamma_{\text {ext }}^{[1]}$ and fulfilling for all $e \in \Gamma^{[1]}$

$$
\bigcup_{j=0}^{1} g^{[0]}\left(\partial_{j}(e)\right)=\bigcup_{j=0}^{1} \partial_{j}\left(g^{[1]}(e)\right) .
$$

Additionally, we require $g^{[0]}$ and $g^{[1]}$ to respect the vertex and edge types given by the sets $\mathcal{R}_{V}$ and $\mathcal{R}_{E}$, respectively.

The automorphism group of $\Gamma$ is denoted by aut $(\Gamma)$ and consists of all such automorphisms of $\Gamma$. The order of the automorphism group of $\Gamma$ is called the symmetry factor of $\Gamma$ and denoted by $\operatorname{sym}(\Gamma)$, i.e.

$$
\operatorname{sym}(\Gamma):=|\operatorname{aut}(\Gamma)| \text {. }
$$

Definition 3.4 (Paths and cycles $[10]$ ). Let $\Gamma$ be a graph, $\Gamma^{[0]}$ its vertex set, $\Gamma^{[1]}$ its edge set and $\gamma$ a subgraph of $\Gamma$. Then:

1. $\gamma$ is called a path (in $\Gamma$ ) if it is non-empty with vertex set $\gamma^{[0]}=\left\{v_{1}, v_{2}, \ldots, v_{v}\right\}\left(\subset \Gamma^{[0]}\right)$ and edge set $\gamma^{[1]}=\left\{v_{1} v_{2}, v_{2} v_{3}, \ldots, v_{v-1} v_{v}\right\}\left(\subset \Gamma^{[1]}\right)$ (where no repeated vertices are allowed, i.e. $v_{i} \neq v_{j}$ for $i \neq j$ ). In particular, a path connects its two endpoints in a unique way and every internal vertex of the path has precisely two edges attached to it. The set of all paths of $\Gamma$ is denoted by $\mathscr{P}(\Gamma)$ and paths therein by $P$.

2. $\gamma$ is called a cycle in mathematics or a loop in physics 5 (of $\Gamma$ ) if it is non-empty with vertex set $\gamma^{[0]}=\left\{v_{1}, v_{2}, \ldots, v_{v}\right\}\left(\subset \Gamma^{[0]}\right)$ and edge set $\gamma^{[1]}=\left\{v_{1} v_{2}, v_{2} v_{3}, \ldots, v_{v-1} v_{v}, v_{v} v_{1}\right\}\left(\subset \Gamma^{[1]}\right)$ (where no repeated vertices are allowed, i.e. $v_{i} \neq v_{j}$ for $i \neq j$ ). In particular, a cycle can be created from the union of two disjoint paths having the same endpoints. The set of all cycles of a graph $\Gamma$ is denoted by $\mathscr{C}(\Gamma)$, a basis of cycles of $\Gamma$ by $\mathfrak{C}(\Gamma)$ and cycles therein by $C$.

Definition 3.5 (Trees, $(n)$-forests and spanning $n$-forests [10]). Let $\Gamma$ be a graph and $\gamma$ a subgraph of $\Gamma$. Then:

1. $\gamma$ is called a forest if it is non-empty and does not contain any cycles. Sets of forests are denoted by $\mathscr{F}$ and forests therein by $F$. If $F$ has $n$ connected components, then $F$ is also called an $n$-forest. Sets of $n$-forests are denoted by $\mathscr{F}_{n}$.

\footnotetext{
${ }^{5} \mathrm{Be}$ aware that a loop in mathematics is what is called a self-loop or a tadpole in physics. We use the terms cycle and loop interchangeably in the above defined sense, depending if the context is more motivated from a mathematical or a physical point of view.
} 
2. If a forest $F$ is connected (i.e. a 1-forest) it is also called a tree. Trees are denoted by $T$ and sets of trees by $\mathscr{T}:=\mathscr{F}_{1}$.

3. If a $n$-forest or a tree is a subgraph of $\Gamma$ and covers all vertices of $\Gamma$, then it is called a spanning $n$-forest or a spanning tree of $\Gamma$, respectively. The sets of all spanning $n$-forests and spanning trees of a graph $\Gamma$ are denoted by $\mathscr{F}_{n}(\Gamma)$ and $\mathscr{T}(\Gamma)$, respectively. In this paper we are only interested in spanning trees and spanning 2-forests of scalar QFT Feynman graphs.

Definition 3.6 ( $k$-Factor [10]). Let $\Gamma$ be a graph and $\gamma$ a subgraph of $\Gamma$. Then:

1. $\gamma$ is called a factor of $\Gamma$, if $\gamma$ covers all vertices of $\Gamma$. The set of all factors of $\Gamma$ is denoted by $\mathfrak{F}(\Gamma)$ and factors therein by 6 .

2. If a factor $\mathfrak{f} \in \mathfrak{F}(\Gamma)$ is $k$-regular, i.e. every vertex of $\mathfrak{f}$ has valence $k$, then $\mathfrak{f}$ is called a $k$-factor of $\Gamma$. The set of $k$-factors for a graph $\Gamma$ is denoted by $\mathfrak{F}_{k}(\Gamma)$.

3. In this paper we are only interested in 2-factors of scalar QFT Feynman graphs. Therefore, we extend the definition of a $k$-factor to the case of graphs with external edges such that external edges also contribute to the valence of the external vertices. Thus, we consider disjoint unions of cycles and paths whose endpoints are external vertices, such that all vertices of the scalar QFT Feynman graph are covered.

Definition 3.7 (Incidence matrix, in parts literally quoted from [2, Page 7]). We choose an (arbitrary) ordering for the edges of our graph $\Gamma$. Then we define the incidence matrix $\varepsilon(\Gamma)$ of a graph $\Gamma$ componentwise as follows:

$$
\varepsilon_{v e}(\Gamma)= \begin{cases}+1 & \text { if the vertex } v \text { is the endpoint of the edge } e \\ -1 & \text { if the vertex } v \text { is the starting point of the edge } e \\ 0 & \text { if } e \text { is not incident to the vertex } v\end{cases}
$$

Definition 3.8 (Assigning 4-vectors to a Feynman graph, in parts literally quoted from [2, Page 7]). We assign a 4 -vector $\xi_{e}^{\prime \mu}$ to each half-edge $h \in \Gamma^{[1 / 2]}$ of a Feynman graph $\Gamma$ in the following way: First we choose a basis of loops $\mathfrak{C}_{\Gamma} \subset \mathscr{C}_{\Gamma}$ for $\Gamma$ and we choose for each $C \in \mathfrak{C}_{\Gamma}$ an orientation $\varepsilon_{v e}^{C}$ (where $\varepsilon_{v e}^{C}$ is defined in such a way that $\varepsilon_{v e_{1}}^{C}=-\varepsilon_{v e_{2}}^{C}$ with $e_{1}$ and $e_{2}$ being two edges adjacent to the vertex $v$ and inside the loop $C$ ). Then we assign to each half-edge $h \equiv(v, e)$ the 4 -vector 7

$$
\varepsilon_{v e} \xi_{e}^{\prime \mu}:=\varepsilon_{v e} \xi_{e}^{\mu}+\sum_{C \in \mathfrak{C}_{\Gamma}} \sum_{e \in C^{[1]}} \varepsilon_{v e}^{C} k_{C}^{\mu}
$$

where the $\left\{\xi_{e}^{\mu}\right\}_{e \in \Gamma^{[1]}}$ are independent in the sense that momentum conservation is not assumed until the end of all calculations (cf. Remark 4.6), and the $\left\{k_{C}^{\mu}\right\}_{C \in \mathfrak{C}_{\Gamma}}$ are the loop momenta which are to be integrated out.

\footnotetext{
${ }^{6}$ Please note the notational difference between a forest of a graph and a factor of a graph.

${ }^{7}$ The notion of the half-edge $h$ is here only important to clarify the orientation of the 4 -vector $\xi_{e}^{\prime \mu}$.
} 
Definition 3.9 (Genus of a graph, in parts literally quoted from [2, Page 4]). Let $\Gamma$ be a graph and $\mathcal{M}_{k}$ an oriented 2-dimensional manifold of genus $k$. Then $\Gamma$ is said to be $k$-compatible, if it can be drawn on $\mathcal{M}_{k}$ without self-intersections. Furthermore, $\Gamma$ is said to be of genus $k$, if it can be drawn on $\mathcal{M}_{k}$ without self intersections, but not on $\mathcal{M}_{l}$ with $l<k$. Planar graphs are of genus 0 .

Definition 3.10 (Orientation of a 3-regular graph, in parts literally quoted from [2, Page 4]). Let $\Gamma$ be a 3-regular $k$-compatible Feynman graph, drawn on an oriented 2-dimensional manifold $\mathcal{M}_{k}$ of genus $k$. Then $\Gamma$ inherits an orientation by $\mathcal{M}_{k}$ which defines to each half-edge $h$ incident to a vertex $v$ a unique successor $h_{+}$and predecessor $h_{-}$.

\section{Parametric Representation of scalar Quantum Field Theories}

The parametric representation for scalar QFTs can be obtained by the use of the so-called Schwinger trick 8 [2, 3, 5, 7]:

$$
\frac{1}{x}=\int_{\mathbb{R}_{+}} e^{-A x} \mathrm{~d} A
$$

Using this trick, the product of propagators in the numerator from the standard Feynman rules can be turned into a sum of an exponential function (where an Euclidean spacetime is assumed and all appearing constants are collected in $\alpha$ ) [3, [5, 7]:

$$
\begin{aligned}
\alpha \prod_{e \in \Gamma_{\text {int }}^{[1]}} \frac{1}{\left(\xi_{e}^{\prime 2}+m_{e}^{2}\right)} & =\alpha \prod_{e \in \Gamma_{\text {int }}^{[1]}} \int_{\mathbb{R}_{+}} e^{-A_{e}\left(\xi_{e}^{\prime 2}+m_{e}^{2}\right)} \mathrm{d} A_{e} \\
& =\alpha \int_{\mathbb{R}_{+}^{\mid \Gamma_{\text {int }}^{[1]}} \mid} e^{-\left(\sum_{e \in \Gamma_{\text {int }}^{[1]}} A_{e}\left(\xi_{e}^{\prime 2}+m_{e}^{2}\right)\right)}\left(\prod_{e \in \Gamma_{\text {int }}^{[1]}} \mathrm{d} A_{e}\right)
\end{aligned}
$$

Remark 4.1. For our purposes in defining the Corolla polynomial in Section 5 we alter the standard definition of the parametric representation of a scalar QFT in two ways: First, we will also include Schwinger variables $A_{e}$ for external edges and secondly we assign 4-vectors $\xi_{e}^{\prime \mu}$ to each edge $e$ of $\Gamma$ which we define to consist of the sum of independent variables $\xi_{e}^{\mu}$ and the corresponding loop momenta $k_{C}^{\mu}$ for $C$ a loop in the basis of loops $\mathfrak{C}_{\Gamma}$ of the Feynman graph $\Gamma$ (cf. Definition 3.8).

Therefore, we define the following simplified notation:

Definition 4.2 (Abbreviations [2]). We denote:

1. The simplex of our parametric integration domain by $\sigma$, i.e.

$$
\sigma:=\mathbb{R}_{+}^{\left|\Gamma^{[1]}\right|}
$$

2. The measure of our extended parametric space by $\mathrm{d} \underline{A}_{\Gamma}$, i.e.

$$
\mathrm{d} \underline{A}_{\Gamma}:=\bigwedge_{e \in \Gamma^{[1]}} \mathrm{d} A_{e} .
$$

\footnotetext{
${ }^{8} \mathrm{~A}$ similar result can be obtained using the so-called Feynman trick 5 .

${ }^{9}$ This can be obtained from the Minkowski spacetime using the so-called Wick rotation [5, 6, 7].
} 
3. The space of all loop momenta by $\rho$ (recall $\left|\mathfrak{C}_{\Gamma}\right|$ to be the dimension of the basis of loops of $\Gamma$ from Definition 3.4 and Definition 3.8), i.e 10

$$
\rho:=\mathbb{R}^{4\left|\mathfrak{C}_{\Gamma}\right|}
$$

4. The measure of the loop momenta integral by $\mathrm{d} \underline{k}_{\Gamma}$ (recall that we choose a basis of loops $\mathfrak{C}_{\Gamma} \subset \mathscr{C}_{\Gamma}$ of $\left|\mathscr{C}_{\Gamma}\right|$ independent loops of $\Gamma$ from Definition 3.8, and $\mathrm{d}^{4} k_{C}$ being the usual Lorentz invariant loop momentum measure for the loop $C$ [5, 6, 7]), i.e 11

$$
\mathrm{d} \underline{k}_{\Gamma}:=\bigwedge_{C \in \mathfrak{C}_{\Gamma}} \mathrm{d}^{4} k_{C}
$$

5. The extended universal quadric by $\underline{Q}_{\Gamma}$ (where the case $m_{e}=0$ for some $e \in \Gamma^{[1]}$ is allowed), i.e.

$$
\underline{Q}_{\Gamma}:=\sum_{e \in \Gamma^{[1]}}\left(\xi_{e}^{\prime 2}+m_{e}^{2}\right) A_{e}
$$

6. The reduced universal quadric by $\underline{q}_{\Gamma}$ (where again the case $m_{e}=0$ for some $e \in \Gamma^{[1]}$ is allowed), i.e.

$$
\underline{q}_{\Gamma}:=\left(\sum_{e \in \Gamma_{\mathrm{ext}}^{[1]}} \xi_{e}^{2} A_{e}\right)+\left(\sum_{e \in \Gamma^{[1]}} m_{e}^{2} A_{e}\right) .
$$

7. The product over the inverse external propagators by $P_{\Gamma}$, i.e.

$$
P_{\Gamma}:=\prod_{e \in \Gamma_{\mathrm{ext}}^{[1]}}\left(\xi_{e}^{2}+m_{e}^{2}\right) .
$$

8. The differential form concerning the parametric space by $I(\Gamma)$, i.e.

$$
I(\Gamma):=\left(\alpha P_{\Gamma} \int_{\rho} e^{-\underline{Q_{\Gamma}}} \prod_{v \in \Gamma^{[0]}} \delta^{(4)}\left(\sum_{e \in \Gamma^{[1]}} \varepsilon_{v e} k_{e}^{\mu}\right) \mathrm{d} \underline{k}_{\Gamma}\right) \mathrm{d} \underline{A}_{\Gamma} .
$$

Then, Equation (10) reads

$$
\alpha \prod_{e \in \Gamma_{\text {int }}^{[1]}} \frac{1}{\left(\xi_{e}^{\prime 2}+m_{e}^{2}\right)}=\alpha P_{\Gamma} \int_{\sigma} e^{-\underline{Q}_{\Gamma}} \mathrm{d} \underline{A}_{\Gamma} .
$$

One of the advantages of passing to the parametric space is that now the loop momentum integrals can be carried out after changing the order of integration 12 . In doing so, the two so-called Kirchhoff- or Symanzik-polynomials $\psi_{\Gamma}$ and $\phi_{\Gamma}[3$ come into play:

\footnotetext{
${ }^{10}$ Note that the dimension is here actually the dimension of the basis of loops of $\Gamma$ times the dimension of spacetime, but we're working in a 4-dimensional spacetime throughout this paper [5, 6, 7.

${ }^{11}$ The power 4 in the measure $\mathrm{d}^{4} k_{C}$ is actually the dimension of spacetime, but again we're working in a 4-dimensional spacetime throughout this paper [5, 6, 7.

${ }^{12}$ The change of the integration order for Minkowski spacetime or massless particles (i.e. ill-defined integral expressions) is formally only allowed if regulators $i \epsilon$ are introduced before in each such propagators and whose limits to 0 are understood to be taken after the integrations are carried out [5, 7].

${ }^{13}$ We will slightly alter the standard definition of the second Symanzik polynomial for our purposes and denote it by $\varphi_{\Gamma}$, cf. Definition 4.5 .
} 
Definition 4.3 (First Symanzik polynomial [2, 3, 5]). Let $\Gamma$ be a scalar QFT Feynman graph and $\mathscr{T}(\Gamma)$ the set of its spanning trees $T$. Then we define the first Symanzik polynomial as (external edges are excluded from the product)

$$
\psi_{\Gamma}:=\sum_{T \in \mathscr{T}(\Gamma)} \prod_{e \notin T} A_{e} .
$$

Example 4.4. We consider the one-loop self-energy graph

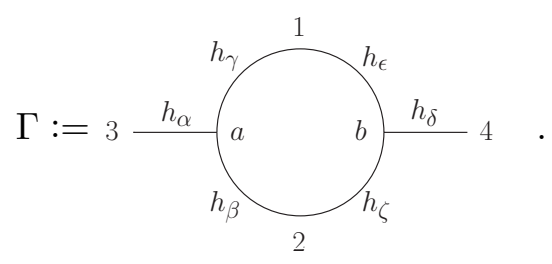

We have $\mathscr{T}(\Gamma)=\{1,2\}$, and so

$$
\psi_{\Gamma}=A_{1}+A_{2}
$$

Definition 4.5 (Second Symanzik polynomial, non-standard definition [2, 3, 5]). Let $\Gamma$ be a scalar QFT Feynman graph, $\mathscr{F}_{2}$ the set of its spanning 2-forests $F$, which consist of the two components $F^{(1)}$ and $F^{(2)}$ (i.e. $F=\bigcup_{i=1}^{2} F^{(i)}$ ), and let $\varepsilon(\Gamma)$ be its incidence matrix (cf. Definition 3.7). Then we define the second Symanzik polynomial as (again, external edges are excluded from the sum and the product)

$$
\varphi_{\Gamma}:=\sum_{F \in \mathscr{F}_{2}(\Gamma)}\left(\sum_{e \notin F} \tau_{F}(e) \xi_{e}^{\mu}\right)^{2} \prod_{e \notin F} A_{e},
$$

with 14

$$
\tau_{F}(e):=-\sum_{v \in F^{(1)}[0]} \varepsilon_{v e}(\Gamma) \equiv \begin{cases}+1 & \text { if } e \text { is oriented from } F^{(1)} \text { to } F^{(2)} \\ -1 & \text { if } e \text { is oriented from } F^{(2)} \text { to } F^{(1)}\end{cases}
$$

Remark 4.6. Note that the usual expression $\phi_{\Gamma}$ for the second Symanzik polynomial of a Feynman graph $\Gamma$ can be obtained by setting the $\left\{\xi_{e}^{\mu}\right\}_{e \in \Gamma^{[1]}}$ in accordance with the physical momenta $\left\{p_{e}^{\mu}\right\}_{e \in \Gamma^{[1]}}$ flowing through the graph (without the loop momenta $\left\{k_{C}^{\mu}\right\}_{C \in \mathfrak{C}_{\Gamma}}$ ) [2, Page 9], i.e.

$$
Q: \quad \xi_{e}^{\mu} \mapsto q_{e}^{\mu}, \quad \forall e \in \Gamma^{[1]} .
$$

Example 4.7. Again, consider the one-loop self-energy graph with incoming external momenta $p^{\mu_{3}}$ and $p^{\mu_{4}}$

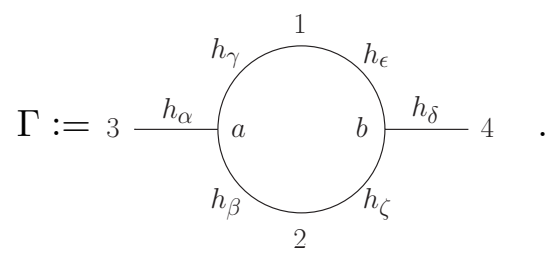

\footnotetext{
${ }^{14}$ Note that if we fix the two components $F^{(1)}$ and $F^{(2)}$ of a spanning 2-forest $F \in \mathscr{F}_{2}(\Gamma)$ and apply momentum conservation as described in Remark [4.6, the standard second Symanzik polynomial becomes independent of that choice since the resulting expressions are squares.
} 
We have $\mathscr{F}_{2}(\Gamma)=\{\{a, b\}\}$, and so

$$
\varphi_{\Gamma}=\left(\xi_{1}^{\mu}-\xi_{2}^{\mu}\right)^{2} A_{1} A_{2} .
$$

Theorem 4.8 (Parametric integrand with non-standard second Symanzik polynomial [2]). Integrating out the loop momenta in the parametric representation gives rise to the two Symanzik polynomial 15 :

$$
\int_{\rho} e^{-\underline{Q}_{\Gamma}} \prod_{v \in \Gamma^{[0]}} \delta^{(4)}\left(\sum_{e \in \Gamma^{[1]}} \varepsilon_{v e} k_{e}^{\mu}\right) \mathrm{d} \underline{k}_{\Gamma}=\frac{e^{-\frac{\varphi_{\Gamma}}{\psi_{\Gamma}}-\underline{q}_{\Gamma}}}{\psi_{\Gamma}^{2}}
$$

In particular, the parametric integrand $I(\Gamma)$ with non-standard second Symanzik polynomial can be written as

$$
\begin{aligned}
I(\Gamma) & =\left(\alpha P_{\Gamma} \int_{\rho} e^{-\underline{Q}_{\Gamma}} \prod_{v \in \Gamma^{[0]}} \delta^{(4)}\left(\sum_{e \in \Gamma^{[1]}} \varepsilon_{v e} k_{e}^{\mu}\right) \mathrm{d} \underline{k}_{\Gamma}\right) \mathrm{d} \underline{A}_{\Gamma} \\
& =\left(\alpha P_{\Gamma} \frac{e^{-\frac{\varphi_{\Gamma}}{\psi_{\Gamma}}-\underline{q}_{\Gamma}}}{\psi_{\Gamma}^{2}}\right) \mathrm{d} \underline{A}_{\Gamma}
\end{aligned}
$$

Proof. We refer to [7, Pages 294 - 299] for a proof of the parametric integrand with standard second Symanzik polynomial and to [2, Page 10] for the notational difference concerning the non-standard second Symanzik polynomial.

Example 4.9. Continuing Example 4.4 and Example 4.7 for the one-loop self-energy graph

$$
\Gamma:=3 \frac{h_{\alpha}}{h_{h_{\beta}}^{h_{\gamma}}} \overbrace{2}^{1} \int_{h_{\zeta}}^{h_{\epsilon}} \frac{h_{\delta}}{a} 4
$$

we have

$$
I(\Gamma)=\left(\left(\xi_{3}^{2}+m_{3}^{2}\right)\left(\xi_{4}^{2}+m_{4}^{2}\right) \frac{e^{-\frac{\left(\xi_{1}^{\mu}-\xi_{2}^{\mu}\right)^{2} A_{1} A_{2}}{A_{1}+A_{2}}-\xi_{3}^{2} A_{3}-\xi_{4}^{2} A_{4}-\sum_{e=1}^{4} m_{e}^{2} A_{e}}}{\left(A_{1}+A_{2}\right)^{2}}\right) \mathrm{d} A_{1} \wedge \mathrm{d} A_{2} .
$$

Definition 4.10 (Parametric integrand for gauge theory amplitudes with non-standard second Symanzik polynomial, in parts literally quoted from [2, Page 10]). In the following we're in particular interested in gauge theory amplitudes. They can be represented in the parametric space by a slightly generalization of $I(\Gamma)$ via

$$
\tilde{I}_{\mathcal{F}}(\Gamma):=\mathcal{F} I(\Gamma),
$$

with

$$
\mathcal{F}:=\frac{\mathcal{F}_{N}\left(\left\{A_{e}\right\}_{e \in \Gamma^{[1]}}\right)}{\mathcal{F}_{D}\left(\left\{A_{e}\right\}_{e \in \Gamma^{[1]}}\right)},
$$

\footnotetext{
${ }^{15}$ The square of $\psi_{\Gamma}$ in the numerator is actually a $d / 2$, with $d$ being the dimension of spacetime, but once more we're working in a 4-dimensional spacetime throughout this paper [3, 5].
} 
a rational function in the Schwinger parameters $A_{e}$ and possible matrix structure. The rational function $F$ can be created by acting with suitable differential operators on the parametric integrand $I(\Gamma)$ and possibly multiply it with the required matrices and the contraction of their respective Lorentz indices [2, 3].

Remark 4.11. Before $I(\Gamma)$ (or $\tilde{I}_{\mathcal{F}}(\Gamma)$ ) can be integrated against the simplex $\sigma$ to yield the Feynman amplitude $\mathcal{I}^{R}(\Gamma)$ (or $\tilde{\mathcal{I}}_{\mathcal{F}}^{R}(\Gamma)$ ) of $\Gamma$ it needs to be renormalized first (cf. [2, 3, 5, 6, 7, 9, 11] for the huge topic of renormalization). The corresponding renormalized differential form $I^{R}(\Gamma)$ (or $\tilde{I}_{\mathcal{F}}^{R}(\Gamma)$ ) can be obtained from $I(\Gamma)\left(\right.$ or $\left.\tilde{I}_{\mathcal{F}}(\Gamma)\right)$ by the use of the Forest formula [2, 3, 5, 17, 11].

\section{Corolla Polynomial and Differential}

\subsection{Pure Yang-Mills Theory}

Now we define the Corolla polynomial for pure Yang-Mills theory:

Definition 5.1 (Corolla polynomial, in parts literally quoted from [1, Definition 1] and [2, Page 27], cf. [3]). Let $\Gamma$ be a 3-regular scalar QFT Feynman graph. Then:

1. Associate to each half-edge $h$ of $\Gamma$ variables $a_{h_{+}}, a_{h_{-}}$and $b_{h}$.

2. Recall from Definition 3.2. For a vertex $v$ of $\Gamma$ we denote the set of the three half-edges incident to $v$ by $n(v)$.

3. Recall from Definition 3.10, For a vertex $v$ of $\Gamma$ and $h \in n(v)$ a half-edge of $\Gamma$, we denote according to the orientation of $\Gamma$ its successor by $h_{+}$and its predecessor by $h_{-}$.

4. We denote the edge $e$ of which $h$ is part of by $e(h)$.

5. Recall from Definition 3.4: We denote the set of all cycles of $\Gamma$ by $\mathscr{C}_{\Gamma}$.

6. For $C \in \mathscr{C}_{\Gamma}$ a cycle and $v$ a vertex in $C$, since $\Gamma$ is 3-regular, there is a unique half-edge of $\Gamma$ incident to $v$ and not in $C$. We denote this half-edge by $h(C, v)$.

7. Assign to $\Gamma$ a factor color $(\Gamma)$.

8. We denote the combination of half-edge variables which will create in the Corolla differential the Feynman rules for the 3 -valent gluon vertex $v$ (and also the 4 -valent gluon vertex as residues in Schwinger parameters if applied twice with special combinations of half-edge variables due to the Leibniz rule, cf. Theorem 5.7 and [2, 3]) as

$$
\mathscr{V}_{v}:=\sum_{h \in n(v)} b_{h}\left(a_{h_{+}}+a_{h_{-}}\right) .
$$

9. We denote the combination of half-edge variables which will create in the Corolla differential the Feynman rules for the closed ghost loop $C_{j}$ as (recall that we work with unoriented ghost edges which means that we sum over both orientations, cf. Subsection 2.3)

$$
\mathscr{G}_{C_{j}}:=\sum_{k \in\{+,-\}} \prod_{v \in C_{j}^{[0]}} a_{h\left(C_{j}, v\right)_{k}} .
$$


Then we can define the various summands of the Corolla polynomial for pure Yang-Mills theory by

$$
\mathcal{C}^{0}(\Gamma):=\prod_{v \in \Gamma^{[0]}} \mathscr{V}_{v}
$$

and for $i \geq 1$ by

$$
\mathcal{C}^{i}(\Gamma):=\sum_{\substack{C_{1}, C_{2}, \ldots, C_{i} \in \mathscr{C}_{\Gamma}, C_{j} \text { pairwise disjoint }}}\left[\left(\prod_{j=1}^{i} \mathscr{G}_{C_{j}}\right)\left(\prod_{\substack{v \in \Gamma^{[0]}, v \notin \bigcup_{k=1}^{i} C_{k}^{[0]}}} \mathscr{V}_{v}\right)\right],
$$

where we define $\prod_{v \in \emptyset} \mathscr{N}_{v}:=1$, i.e. if $\left\{v \in \Gamma^{[0]} \mid v \notin \bigcup_{k=1}^{i} C_{k}\right\}=\emptyset$ (all vertices are attached to edges that will be turned into a ghost edges). Finally, we introduce the Corolla polynomial as the alternating sum over its summands:

$$
\mathcal{C}(\Gamma):=\sum_{i=1}^{\infty}(-1)^{i} \mathcal{C}^{i}(\Gamma)
$$

Furthermore, we define

$$
\mathcal{C}_{\mathrm{QCD}}(\Gamma):=i^{\left|\Gamma^{[1]}\right|} \underline{g}^{\mid \Gamma^{[0]}} \mid \operatorname{color}(\Gamma) \mathcal{C}(\Gamma)
$$

Remark 5.2. Note that our definition of the Corolla polynomial in the half-edge variables $\left\{a_{h_{+}}, a_{h_{-}}, b_{h}\right\}_{h \in \Gamma^{[1 / 2]}}$ differs from the ones given in [1, 2, 3], but we will also define different differential operators $\left\{\mathscr{A}_{h_{+}}, \mathscr{A}_{h_{-}}\right\}_{h \in \Gamma^{[1 / 2]}}$ and metric tensors $\left\{\mathscr{B}_{h}\right\}_{h \in \Gamma^{[1 / 2]}}$ such that the Corolla differentials $\mathcal{D}(\Gamma)$ coincide again (despite from factors of $1 / 4^{i}$ which were missing in each summand $\mathcal{D}^{i}(\Gamma)$ in [1, 2], cf. [3] and the fact that our definition does not create 4-valent ghost vertex contributions from the start which need to be eliminated in the formulation of [1, 2, 3] while working in a linear covariant gauge otherwise, cf. [2]). Furthermore, the above formulation, originating from [4], is only valid for the Feynman gauge (which is in particular linear). This simplifies the numerator of gauge boson propagators to metric tensors, cf. [2] for the general case. Together with our extended definition of the Einstein summation convention in Subsection 2.1 we can effectively work with scalar QFT propagators since all relevant quantum gauge theory tensor structures are created from the corresponding vertex Feynman rules.

Remark 5.3. $\mathcal{C}(\Gamma)$ is a polynomial since $\mathcal{C}^{i}(\Gamma)=0$ for all $i>\left|\mathfrak{C}_{\Gamma}\right|$ [1, 2].

Example 5.4. Consider the one-loop self-energy graph

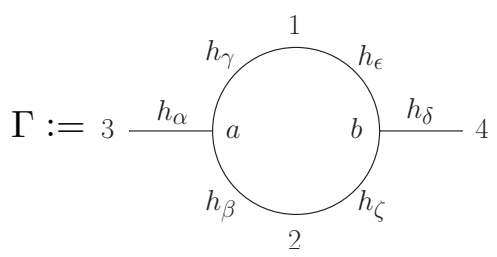

with the six half-edges $\left\{h_{\alpha}:=(a, 3), h_{\beta}=(a, 2), h_{\gamma}=(a, 1), h_{\delta}=(b, 4), h_{\epsilon}=(b, 1), h_{\zeta}=(b, 2)\right\}$. 
Then, we have

$$
\begin{gathered}
\mathcal{C}^{0}(\Gamma)=\left(b_{h_{\alpha}}\left(a_{h_{\alpha+}}+a_{h_{\alpha-}}\right)+b_{h_{\beta}}\left(a_{h_{\beta+}}+a_{h_{\beta-}}\right)+b_{h_{\gamma}}\left(a_{h_{\gamma+}}+a_{h_{\gamma-}}\right)\right) \\
\times\left(b_{h_{\delta}}\left(a_{h_{\delta+}}+a_{h_{\delta-}}\right)+b_{h_{\epsilon}}\left(a_{h_{\epsilon+}}+a_{h_{\epsilon-}}\right)+b_{h_{\zeta}}\left(a_{h_{\zeta+}}+a_{h_{\zeta-}}\right)\right), \\
\mathcal{C}^{1}(\Gamma)=a_{h_{\alpha+}} a_{h_{\delta+}}+a_{h_{\alpha-}} a_{h_{\delta-}}
\end{gathered}
$$

and

$$
\mathcal{C}^{i}(\Gamma)=0, \quad \forall i>1
$$

So, in total we get:

$$
\begin{aligned}
\mathcal{C}(\Gamma)= & \mathcal{C}^{0}(\Gamma)-\mathcal{C}^{1}(\Gamma) \\
= & \left(b_{h_{\alpha}}\left(a_{h_{\alpha+}}+a_{h_{\alpha-}}\right)+b_{h_{\beta}}\left(a_{h_{\beta+}}+a_{h_{\beta-}}\right)+b_{h_{\gamma}}\left(a_{h_{\gamma+}}+a_{h_{\gamma-}}\right)\right) \\
& \times\left(b_{h_{\delta}}\left(a_{h_{\delta+}}+a_{h_{\delta-}}\right)+b_{h_{\epsilon}}\left(a_{h_{\epsilon+}}+a_{h_{\epsilon-}}\right)+b_{h_{\zeta}}\left(a_{h_{\zeta+}}+a_{h_{\zeta-}}\right)\right) \\
& -a_{h_{\alpha+}} a_{h_{\delta+}}-a_{h_{\alpha-}} a_{h_{\delta-}}
\end{aligned}
$$

Definition 5.5 (Corolla differential, in parts literally quoted from [2, Page 29]). Let $\Gamma$ be a 3-regular scalar QFT Feynman graph. Then:

1. Assign to each external and internal edge a variable $A_{e}$ and a 4 -vector $\xi_{e}^{\prime \mu}$ (as in Definition 3.8) and to each edge $e$ a Lorentz index $\mu_{e}$.

2. Define for each half-edge $h_{k}$ the following differential operator (where $k \in\{ \pm\}$ ):

$$
\mathscr{A}_{h_{k}}:=-k \varepsilon_{h_{k}} \frac{1}{2 A_{e\left(h_{k}\right)}} \frac{\partial}{\partial \xi_{e\left(h_{k}\right) \mu_{e(h)}}}
$$

3. Define for each half-edge $h$ the following metric tensor:

$$
\mathscr{B}_{h}:=\eta^{\mu}{ }^{\mu\left(h_{+}\right)^{\mu}} e_{\left(h_{-}\right)}
$$

Then, the summands of the Corolla differential $\mathcal{D}^{i}(\Gamma)$ are defined via the summands of the Corolla polynomial $\mathcal{C}^{i}(\Gamma)$ by replacing each half-edge variable $a_{h_{k}}$ by the corresponding differential operator $\mathscr{A}_{h_{k}}$ (denoted by $a_{h_{k}} \mapsto \mathscr{A}_{h_{k}}$ ) and each half-edge variable $b_{h}$ by the corresponding metric tensor $\mathscr{B}_{h}$ (denoted by $b_{h} \mapsto \mathscr{B}_{h}$ ):

$$
\mathcal{D}^{i}(\Gamma):=\left.\mathcal{C}^{i}(\Gamma)\right|_{\substack{a_{h_{k}} \mapsto \mathscr{A}_{h_{k}}, \forall h_{k} \in \Gamma^{[1 / 2]} \\ b_{h} \mapsto \mathscr{B}_{h}, \forall h \in \Gamma^{[1 / 2]}}},
$$

and similarly the Corolla differential $\mathcal{D}(\Gamma)$ is defined like the Corolla polynomial $\mathcal{C}(\Gamma)$ as

$$
\mathcal{D}(\Gamma):=\left.\sum_{i=1}^{\infty}(-1)^{i} \mathcal{D}^{i}(\Gamma) \equiv \mathcal{C}(\Gamma)\right|_{\begin{array}{c}
a_{h_{k}} \mapsto \mathscr{A}_{h_{k}}, \forall h_{k} \in \Gamma^{[1 / 2]} \\
b_{h} \mapsto \mathscr{B}_{h}, \forall h \in \Gamma^{[1 / 2]}
\end{array}} .
$$

Likewise, the Corolla differentials $\mathcal{D}_{\mathrm{QCD}}(\Gamma)$ and $\mathcal{D}_{\mathrm{EW}}(\Gamma)$ are defined via the replacement of the half-edge variables $a_{h_{k}}$ by the corresponding differential operators $\mathscr{A}_{h_{k}}$ and the half-edge variables $b_{h}$ by the corresponding metric tensors $\mathscr{B}_{h}$ in their corresponding Corolla polynomials $\mathcal{C}_{\mathrm{QCD}}(\Gamma)$ and $\mathcal{C}_{\mathrm{EW}}(\Gamma)$ (the latter one will be defined in Definition [5.19), respectively. 
Remark 5.6. 1. Expressions of the form $\mathcal{D}(\Gamma) I(\Gamma)$ are to be understood a: 16

$$
\mathcal{D}(\Gamma) I(\Gamma):=\left.(\mathcal{D}(\Gamma) I(\Gamma))\right|_{\xi_{e}^{\mu} \mapsto q_{e}^{\mu}, \forall e \in \Gamma^{[1]}},
$$

such that the Corolla differential acts on the parametric integrand with non-standard second Symanzik polynomial (cf. Definition 4.5 and Theorem 4.8) and after the differentiation the standard second Symanzik polynomial is obtained by setting the $\left\{\xi_{e}^{\mu}\right\}_{e \in \Gamma^{[1]}}$ in accordance with the external momenta (cf. Remark 4.6). Note that due to the Leibniz rule we get also contributions from differentiating the inverse external propagators $P_{\Gamma}$ (cf. 7. from Definition 4.2) but since the differential operators for the external edges are of order 1 , these contributions vanish again whilst setting the $\left\{\xi_{e}^{\mu}\right\}_{e \in \Gamma^{[1]}}$ in accordance with the external momenta (again, cf. Remark 4.6).

2. We choose the 4 -vectors $\left\{\xi_{e}^{\mu}\right\}_{e \in \Gamma^{[1]}}$ assigned to each edge of the graph independently, i.e. we do not assume momentum conservation until the Corolla differential acted on the scalar integrand (cf. Definition 3.8). Therefore, they can be seen as "temporary dummy variables". After applying the Corolla differential, we assume momentum conservation and they acquire the meaning of physical 4-momentum vectors of the momenta flowing through the graph (without the loop momenta $\left\{k_{C}^{\mu}\right\}_{C \in \mathfrak{C}_{\Gamma}}$ ) and are then denoted as $\left\{q_{e}^{\mu}\right\}_{e \in \Gamma^{[1]}}$.

3. $\mathcal{D}^{i}(\Gamma)$ creates, whilst acting on the corresponding parametric scalar QFT integrand $I(\Gamma)$, all possible pure Yang-Mills theory Feynman graphs with $\left|\mathfrak{C}_{\Gamma}\right|$ loops and $i$ ghost loops. The alternating sum in the Corolla polynomial $\mathcal{C}(\Gamma)$ and hence also in the Corolla differential $\mathcal{D}(\Gamma)$ takes care of the minus sign for each closed ghost loop (cf. Theorem 5.7).

Theorem $5.7([2])$. Using the above definitions, the renormalized amplitude $\tilde{\mathcal{I}}_{\mathcal{F}}^{R}(\Gamma)$ of all gauge theory graphs of pure Yang-Mills theory which can be achieved from $\Gamma$ via graph and cycle homology [2] is then obtained via the Corolla differential $\mathcal{D}_{\mathrm{QCD}}(\Gamma)$ (cf. Definition 5.5) and the renormalized parametric integrand with non-standard second Symanzik polynomial $I^{R}(\Gamma)$ (cf. Theorem 4.8 and again [2, 3, 5, 6, 79, 9, 11] for the huge topic of renormalization)

$$
\tilde{I}_{\mathcal{F}}^{R}(\Gamma)=\mathcal{D}_{\mathrm{QCD}}(\Gamma) I^{R}(\Gamma)
$$

by (where the hat over a variable $\widehat{A_{e}}$ means that we omit this particular variable $A_{e}$ )

$$
\tilde{\mathcal{I}}_{\mathcal{F}}^{R}(\Gamma)=\frac{1}{\operatorname{sym}(\Gamma)} \sum_{k=0}^{\infty} \sum_{\left\{e_{1}, \ldots, e_{k}\right\} \subset \Gamma_{\text {int }}^{[1]}} \int \mathrm{d} \underline{A}_{\Gamma \backslash\left\{e_{1}, \ldots, e_{k}\right\}} \underset{A_{1}, \ldots, \widehat{A_{e_{1}}}, \ldots, \widehat{A_{e_{k}}, \ldots, A_{e} \Gamma^{[1]} \mid}=0}{\operatorname{Reg}} \underset{A_{e_{1}}, \ldots, A_{e_{k}}=0}{\operatorname{Res}} \tilde{I}_{\mathcal{F}}^{R}(\Gamma) .
$$

Proof. We refer to [2, Page 31] for a proof.

Example 5.8. We continue with Example 5.4:

Again, we have

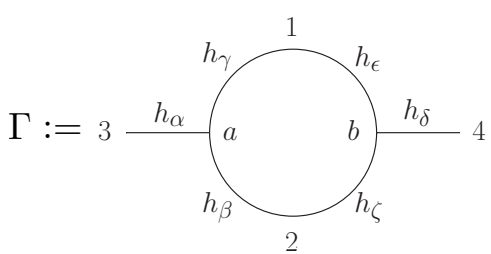

\footnotetext{
${ }^{16}$ Where the $\left\{q_{e}^{\mu}\right\}_{e \in \Gamma^{[1]}}$ are the physical 4-momenta of the scalar QFT Feynman graph $\Gamma$, i.e. the physical momenta flowing through the graph (without the loop momenta $\left\{k_{C}^{\mu}\right\}_{C \in \mathfrak{C}_{\Gamma}}$ )
} 
and

$$
\begin{aligned}
\mathcal{C}(\Gamma)= & \mathcal{C}^{0}(\Gamma)-\mathcal{C}^{1}(\Gamma) \\
= & \left(b_{h_{\alpha}}\left(a_{h_{\alpha+}}+a_{h_{\alpha-}}\right)+b_{h_{\beta}}\left(a_{h_{\beta+}}+a_{h_{\beta-}}\right)+b_{h_{\gamma}}\left(a_{h_{\gamma+}}+a_{h_{\gamma-}}\right)\right) \\
& \times\left(b_{h_{\delta}}\left(a_{h_{\delta+}}+a_{h_{\delta-}}\right)+b_{h_{\epsilon}}\left(a_{h_{\epsilon+}}+a_{h_{\epsilon-}}\right)+b_{h_{\zeta}}\left(a_{h_{\zeta+}}+a_{h_{\zeta-}}\right)\right) \\
& -a_{h_{\alpha+}} a_{h_{\delta+}}-a_{h_{\alpha-}} a_{h_{\delta-}}
\end{aligned}
$$

Choosing an anti-clockwise orientation for our embedding oriented manifold, external momenta $p^{\mu_{3}}$ and $p^{\mu_{4}}$ incoming and also an anti-clockwise orientation for the loop momenta, we receive:

$$
\begin{aligned}
& \mathscr{A}_{h_{\alpha+}}:=\frac{1}{2 A_{2}} \frac{\partial}{\partial \xi_{2 \mu_{3}}}, \quad \mathscr{A}_{h_{\alpha-}}:=\frac{1}{2 A_{1}} \frac{\partial}{\partial \xi_{1 \mu_{3}}}, \mathscr{A}_{h_{\beta-}}:=\frac{1}{2 A_{3}} \frac{\partial}{\partial \xi_{3 \mu_{2}}}, \\
& \mathscr{A}_{h_{\beta+}}:=-\frac{1}{2 A_{1}} \frac{\partial}{\partial \xi_{1 \mu_{2}}}, \quad \mathscr{A}_{h_{\gamma-}}:=-\frac{1}{2 A_{2}} \frac{\partial}{\partial \xi_{2 \mu_{1}}},=-\frac{1}{2 A_{3}} \frac{\partial}{\partial \xi_{3 \mu_{1}}}, \quad \mathscr{A}_{h_{\delta-}}:=\frac{1}{2 A_{2}} \frac{\partial}{\partial \xi_{2 \mu_{4}}}, \\
& \mathscr{A}_{h_{\delta+}}:=\frac{1}{2 A_{1}} \frac{\partial}{\partial \xi_{1 \mu_{4}}}, \quad \mathscr{A}_{h_{\epsilon-}}:=\frac{1}{2 A_{4}} \frac{\partial}{\partial \xi_{4 \mu_{1}}}, \\
& \mathscr{A}_{h_{\epsilon+}}:=-\frac{1}{2 A_{2}} \frac{\partial}{\partial \xi_{2 \mu_{1}}}, \quad \mathscr{A}_{h_{\zeta-}}:=-\frac{1}{2 A_{1}} \frac{\partial}{\partial \xi_{1 \mu_{2}}} \\
& \mathscr{A}_{h_{\zeta+}}:=-\frac{1}{2 A_{4}} \frac{\partial}{\partial \xi_{4 \mu_{2}}}, \\
& \mathscr{B}_{h_{\alpha}}:=\eta^{\mu_{2} \mu_{1}}, \\
& \mathscr{B}_{h_{\beta}}:=\eta^{\mu_{1} \mu_{3}} \\
& \mathscr{B}_{h_{\gamma}}:=\eta^{\mu_{3} \mu_{2}}, \\
& \mathscr{B}_{h_{\delta}}:=\eta^{\mu_{1} \mu_{2}}, \\
& \mathscr{B}_{h_{\epsilon}}:=\eta^{\mu_{2} \mu_{4}}, \\
& \mathscr{B}_{h_{\zeta}}:=\eta^{\mu_{4} \mu_{1}}
\end{aligned}
$$

and

$$
\begin{aligned}
\mathcal{D}(\Gamma)= & \left(\mathscr{B}_{h_{\alpha}}\left(\mathscr{A}_{h_{\alpha+}}+\mathscr{A}_{h_{\alpha-}}\right)+\mathscr{B}_{h_{\beta}}\left(\mathscr{A}_{h_{\beta+}}+\mathscr{A}_{h_{\beta-}}\right)+\mathscr{B}_{h_{\gamma}}\left(\mathscr{A}_{h_{\gamma+}}+\mathscr{A}_{h_{\gamma-}}\right)\right) \\
& \times\left(\mathscr{B}_{h_{\delta}}\left(\mathscr{A}_{h_{\delta+}}+\mathscr{A}_{h_{\delta-}}\right)+\mathscr{B}_{h_{\epsilon}}\left(\mathscr{A}_{h_{\epsilon+}}+\mathscr{A}_{h_{\epsilon-}}\right)+\mathscr{B}_{h_{\zeta}}\left(\mathscr{A}_{h_{\zeta+}}+\mathscr{A}_{h_{\zeta-}}\right)\right) \\
& -\mathscr{A}_{h_{\alpha+}} \mathscr{A}_{h_{\delta+}}-\mathscr{A}_{h_{\alpha}} \mathscr{A}_{h_{\delta-}} \\
= & \left(\eta^{\mu_{2} \mu_{1}}\left(\frac{1}{2 A_{2}} \frac{\partial}{\partial \xi_{2 \mu_{3}}}+\frac{1}{2 A_{1}} \frac{\partial}{\partial \xi_{1 \mu_{3}}}\right)+\eta^{\mu_{1} \mu_{3}}\left(-\frac{1}{2 A_{1}} \frac{\partial}{\partial \xi_{1 \mu_{2}}}+\frac{1}{2 A_{3}} \frac{\partial}{\partial \xi_{3 \mu_{2}}}\right)\right. \\
& \left.+\eta^{\mu_{3} \mu_{2}}\left(-\frac{1}{2 A_{3}} \frac{\partial}{\partial \xi_{3 \mu_{1}}}-\frac{1}{2 A_{2}} \frac{\partial}{\partial \xi_{2 \mu_{1}}}\right)\right) \times\left(\eta^{\mu_{1} \mu_{2}}\left(\frac{1}{2 A_{1}} \frac{\partial}{\partial \xi_{1 \mu_{4}}}+\frac{1}{2 A_{2}} \frac{\partial}{\partial \xi_{2 \mu_{4}}}\right)\right. \\
& \left.+\eta^{\mu_{2} \mu_{4}}\left(-\frac{1}{2 A_{2}} \frac{\partial}{\partial \xi_{2 \mu_{1}}}+\frac{1}{2 A_{4}} \frac{\partial}{\partial \xi_{4 \mu_{1}}}\right)+\eta^{\mu_{4} \mu_{1}}\left(-\frac{1}{2 A_{4}} \frac{\partial}{\partial \xi_{4 \mu_{2}}}-\frac{1}{2 A_{1}} \frac{\partial}{\partial \xi_{1 \mu_{2}}}\right)\right) \\
& -\frac{1}{4 A_{2} A_{1}} \frac{\partial}{\partial \xi_{2 \mu_{3}}} \frac{\partial}{\partial \xi_{1 \mu_{4}}}-\frac{1}{4 A_{1} A_{2}} \frac{\partial}{\partial \xi_{1 \mu_{3}}} \frac{\partial}{\partial \xi_{2 \mu_{4}}} .
\end{aligned}
$$


Acting with this differential operator on the scalar QFT parametric integrand with nonstandard second Symanzik polynomial $I(\Gamma)$ (cf. Example 4.9), we receive [3, Example 5.15]

$$
\begin{aligned}
\tilde{I}_{\mathcal{F}}(\Gamma)= & \mathcal{D}_{\mathrm{QCD}}(\Gamma) I(\Gamma) \\
=\underline{g_{s}}{ }^{2} f^{c_{3} c_{2} c_{1}} f^{c_{4} c_{2} c_{1}}( & \left(q^{\mu_{3}} q^{\mu_{4}}\left(2 A_{1}^{2}+2 A_{2}^{2}+12 A_{1} A_{2}\right)\right. \\
& \left.\left.\quad-q^{2} \eta^{\mu_{3} \mu_{4}}\left(5 A_{1}^{2}+5 A_{2}^{2}+8 A_{1} A_{2}\right)\right) \frac{1}{\psi_{\Gamma}^{2}} 8+\eta^{\mu_{3} \mu_{4}} \frac{1}{\psi_{\Gamma}}\right) I(\Gamma) .
\end{aligned}
$$

Then, we obtain (after renormalizing the parametric integrand $I(\Gamma)$ ) the renormalized Feynman amplitude with renormalization point $\mu$ [3, Example 5.15]

$$
\tilde{\mathcal{I}}_{\mathcal{F}}^{R}(\Gamma)=\frac{10}{3} \underline{g}_{s}^{2} f^{c_{3} c_{2} c_{1}} f^{c_{4} c_{2} c_{1}}\left(-q^{\mu_{3}} q^{\mu_{4}}+q^{2} \eta^{\mu_{3} \mu_{4}}\right) \ln \frac{q^{2}}{\mu^{2}}
$$

which is transversal, as desired.

Remark 5.9. The full $m$-loop scattering amplitude can be obtained by applying the Corolla polynomial componentwise to the combinatorial Green's function for connected graph $\$ 17$, with loop order $m[2]$.

\subsection{Inclusion of the Gauge Bosons of the Electroweak Sector}

The inclusion of the gauge bosons and their corresponding ghosts of the electroweak sector of the Standard Model shows some interesting properties and is therefore the next step to adapt the Corolla polynomial to all bosons of the electroweak sector of the Standard Model. Concretely, we have to add the massive gauge bosons $W^{ \pm}$and $Z$ with their corresponding ghosts $c^{ \pm}$and $c_{Z}$ and the photon $A$ with its corresponding ghost $c_{A}$.

Outline. First, recall that we have $W^{ \pm}$-particle conservation which implies that every vertex of the electroweak sector of the Standard Model has to consist of a positive even number of $W^{ \pm}$-particles, in particular every 3 -valent vertex must consist of two $W^{ \pm}$-particles. This is precisely the definition of a 2-factor, extended to external edges of a scalar QFT Feynman graph. Therefore, we define two nested sums over a 3-regular scalar QFT Feynman graph $\Gamma$ in the following way: The first sum runs over all 2 -factors of $\Gamma$, thus creating all possible ways to attach valid $W^{ \pm}$-particle labelings to $\Gamma$. Then, the second sum creates all possible ways to attach $Z$-labels or $A$-labels to the unlabeled edges of the $W^{ \pm}$-labeled graphs. The full details are explained in the proof after Theorem 5.12 .

Therefore, we define:

Definition 5.10. Let $\Gamma$ be a 3-regular graph. Then:

1. Let $\wp\left(\Gamma^{[1]}\right)$ be the power set of all external edges and internal edges of $\Gamma$ with all adjacent vertices added. In particular, we define $18 \mathscr{P}_{Z}(\mathfrak{f}):=\wp\left(\Gamma^{[1]} \backslash \mathfrak{f}^{[1]}\right)$, i.e. the power set of all external edges and internal edges of $\Gamma$ which are not in the set $\mathfrak{f}$. We denote the elements of $\mathscr{P}_{Z}(\mathfrak{f})$ by $P_{Z}$. The edges in each set $P_{Z}$ in the set of sets $\mathscr{P}_{Z}(\mathfrak{f})$ are labeled by a $Z$-label and the edges in $\Gamma \backslash\left(\mathfrak{f} \cup P_{Z}\right)$ are labeled by an $A$-label.

\footnotetext{
${ }^{17}$ We emphasize that we really mean 1-connected graphs, not only 2-connected (as they are called in mathematics) or 1 PI (as they are called in physics) graphs.

${ }^{18}$ Recall Definition 3.6 for the definition of a 2-factor of a scalar QFT Feynman graph.
} 
2. Let $\left|P_{Z}^{[0]}\right|$ and $\left|\Gamma^{[0]} \backslash P_{Z}^{[0]}\right|$ denote the number of vertices in $P_{Z}$ and $\Gamma \backslash P_{Z}$, respectively.

3. Let iso $\left(\Gamma_{\text {labeled }}\right)$ be the number of labeled graphs (via the sets $\mathfrak{f}$ and $P_{Z}$ ) in the set $\left\{P_{Z} \in \mathscr{P}_{Z}(\mathfrak{f}) \mid \mathfrak{f} \in \mathfrak{F}_{2}(\Gamma)\right\}$ isomorphic to $\Gamma_{\text {labeled }}$.

Example 5.11. In particular, for the one-loop self-energy graph

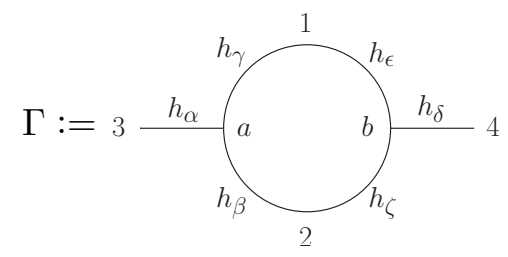

we have:
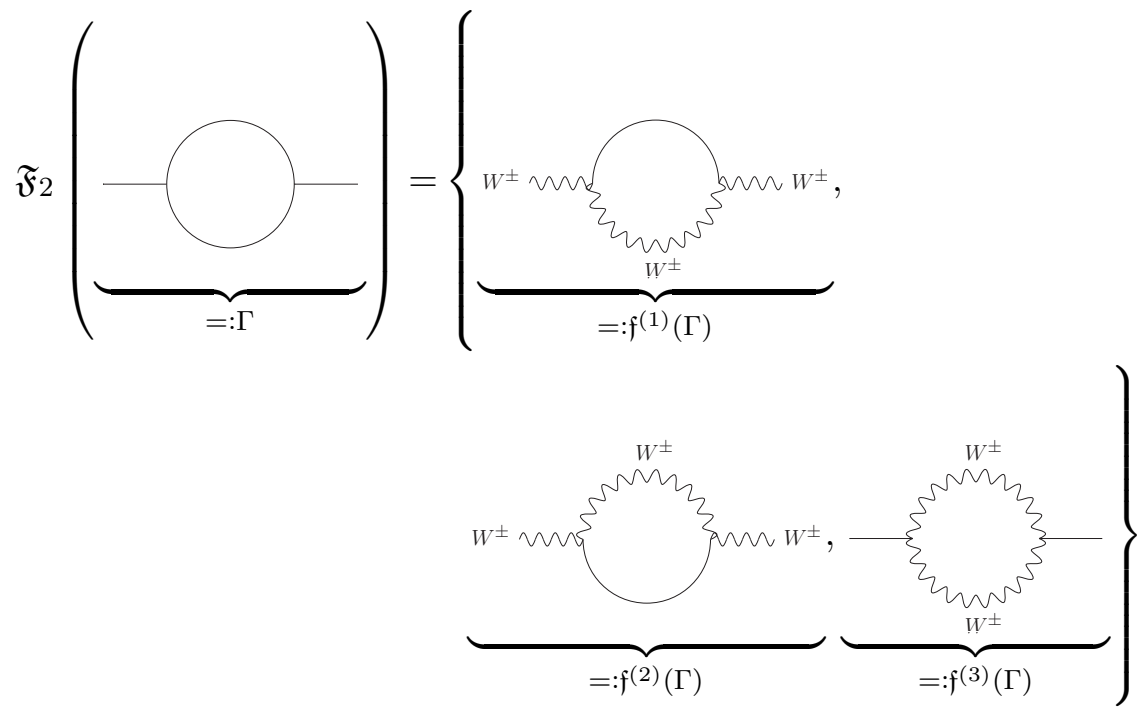

and
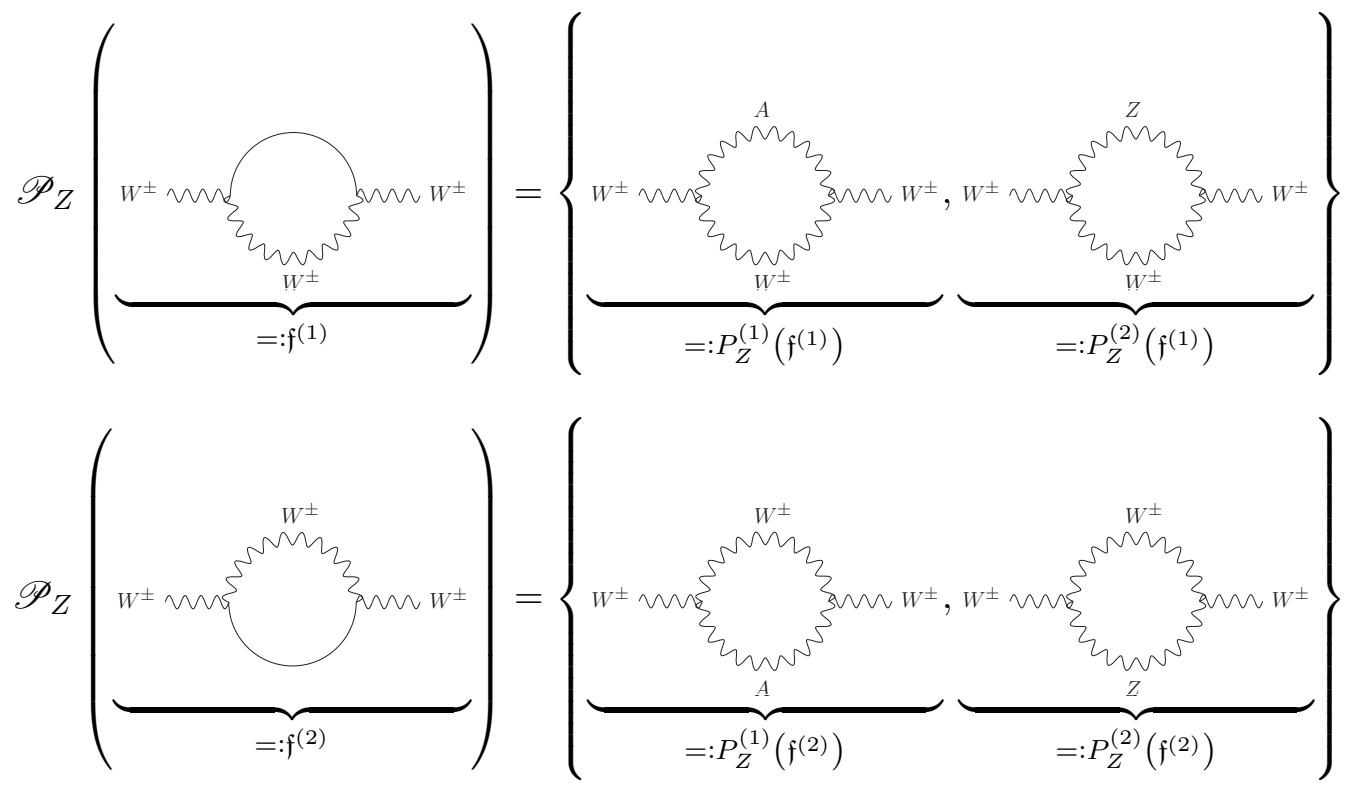

(41b) 

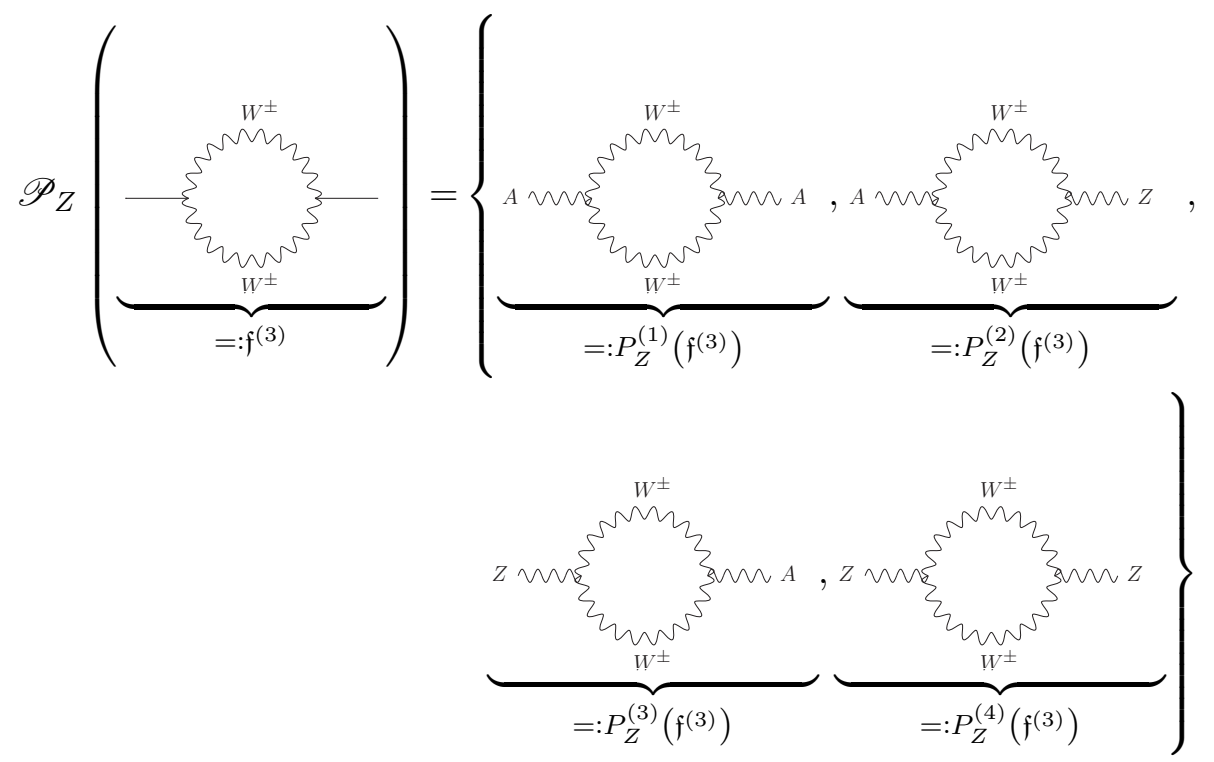

Moreover, the above defined factors read
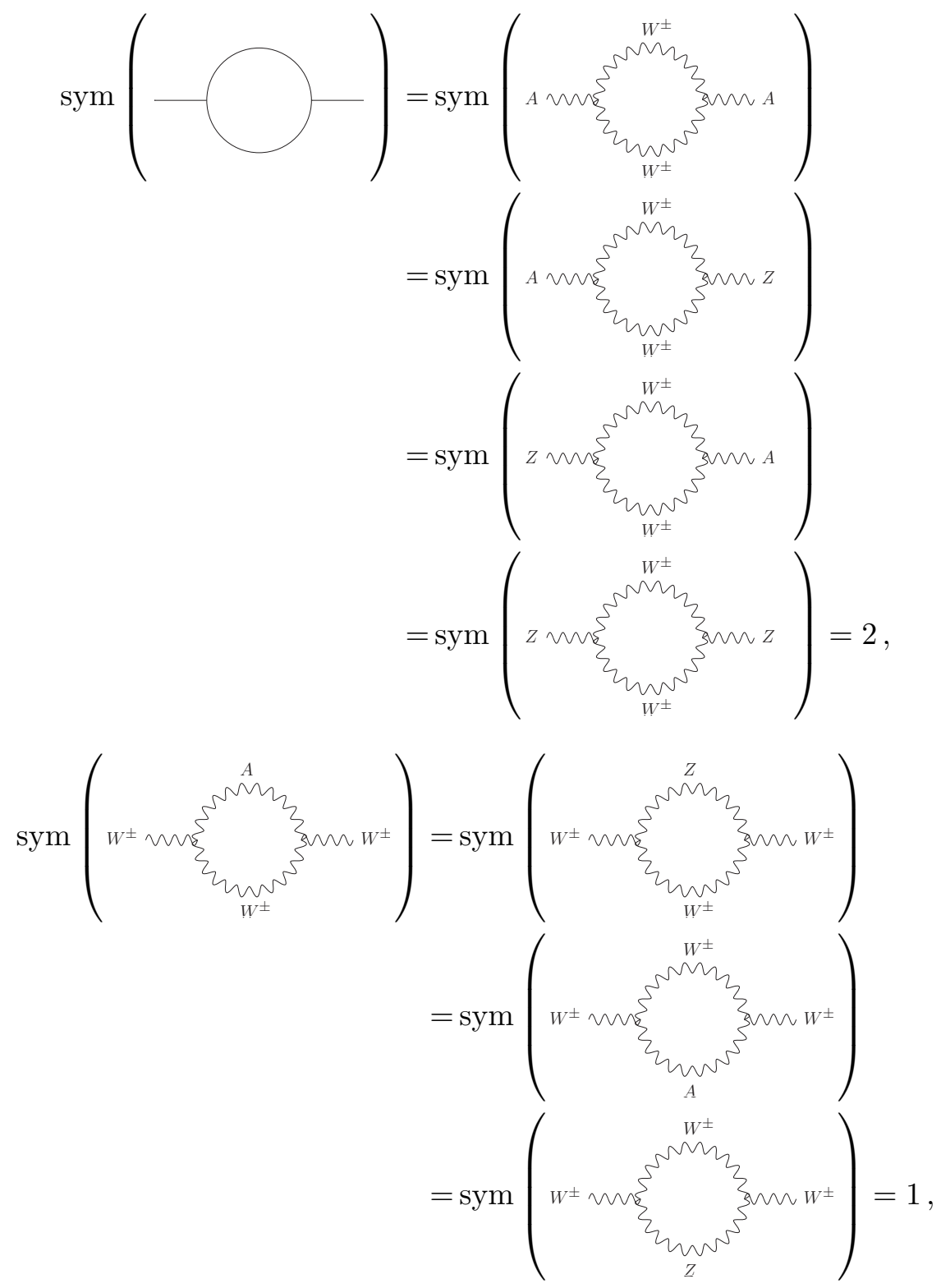


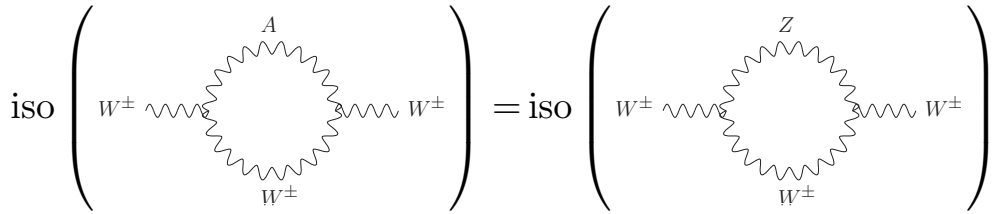

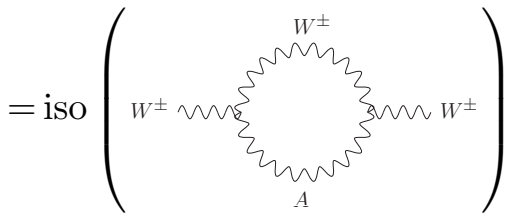

$$
\begin{aligned}
& =\text { iso }\left(W^{ \pm} m \sum_{7}^{W^{ \pm}} \xi^{2} \sin W^{ \pm}\right)=2
\end{aligned}
$$

and

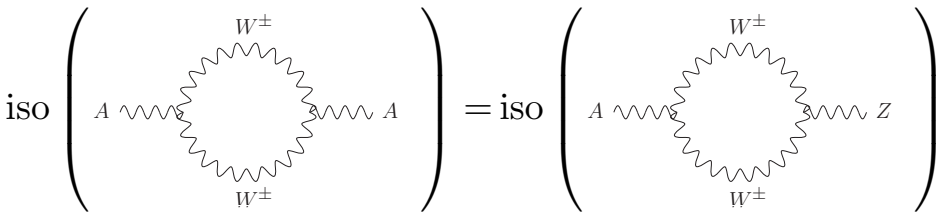

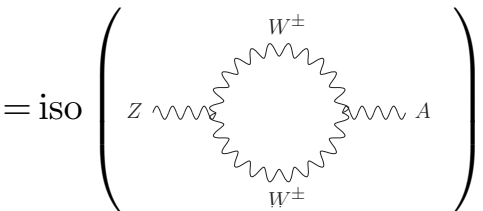

$$
\begin{aligned}
& =\operatorname{iso}\left(z m \sum_{W^{ \pm}}^{W^{ \pm}} \xi^{W^{ \pm}} \operatorname{smz} z\right)=1 \text {, }
\end{aligned}
$$

such that

$$
\frac{\operatorname{sym}(\Gamma)}{\operatorname{sym}\left(\Gamma_{\text {labeled }}\right) \text { iso }\left(\Gamma_{\text {labeled }}\right)}=1, \quad \forall P_{Z} \in \mathscr{P}_{Z}(\mathfrak{f}), \quad \forall \mathfrak{f} \in \mathfrak{F}_{2}(\Gamma),
$$

where we remind the reader that we consider the one-loop self-energy graph

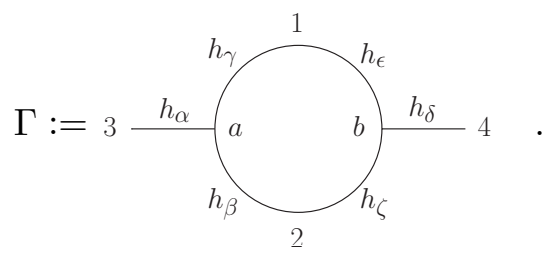

Then, we have:

Theorem 5.12 ([4]). Let $\Gamma$ be a 3-regular scalar QFT Feynman graph. Then the gauge bosons and their corresponding ghosts of the electroweak sector of the Standard Model can be included into the definition of the Corolla polynomial (Definition 5.1) by multiplying the parametric in- 
tegrand $I(\Gamma)$ of the corresponding scalar QFT amplitude by $J\left(\Gamma ; m_{W}, m_{Z}\right)$, defined as

$$
\begin{aligned}
J\left(\Gamma ; m_{W}, m_{Z}\right):= & \left(i^{\left|\Gamma^{[1]}\right|} \underline{\underline{g}}^{\left|\Gamma^{[0]}\right|} \operatorname{color}(\Gamma)\right. \\
& +\sum_{\mathfrak{f} \in \mathfrak{F}_{2}(\Gamma) P_{Z} \in \mathscr{P}_{Z}(\mathfrak{f})} \frac{\operatorname{sym}(\Gamma)}{\operatorname{sym}\left(\Gamma_{\text {labeled }}\right) \text { iso }\left(\Gamma_{\text {labeled }}\right)} \\
& \left.\times i^{\left|\Gamma^{[0]}\right|+\left|\Gamma^{[1]}\right|}\left(\underline{g} \cos \theta_{W}\right)^{\left|P_{Z}^{[0]}\right|} \underline{e}^{\left|\Gamma^{[0]} \backslash P_{Z}^{[0]}\right|} e^{-\left(\sum_{e \in \mathfrak{f}}^{[1]} A_{e} m_{W}^{2}+\sum_{e \in P_{Z}^{[1]}} A_{e} m_{Z}^{2}\right)}\right),
\end{aligned}
$$

such that the corresponding parametric integrand for gauge theory including gluons, ghosts, electroweak gauge bosons and their corresponding ghosts $\tilde{I}_{\mathcal{F}}(\Gamma)$ can be written a 19

$$
\tilde{I}_{\mathcal{F}}(\Gamma)=\mathcal{D}(\Gamma) I(\Gamma) J\left(\Gamma ; m_{W}, m_{Z}\right) .
$$

Proof. Recall that the Feynman rules do not allow gluons to couple to the gauge bosons of the electroweak sector of the Standard Model. Therefore, we can consider these two parts of the Standard Model separately. Additionally note that the Feynman rules for the gauge bosons of the electroweak sector of the Standard Model have precisely the same tensor structure as the Feynman rules for the gluons. The only difference lies in different factors of $i$, different coupling constants, the structure constants for the gluons and the mass terms for the massive gauge bosons and their corresponding ghosts of the electroweak sector of the Standard Model. These are taken in account by the factors $i^{\left|\Gamma^{[1]}\right|} \underline{g s}^{\left|\Gamma^{[0]}\right|} \operatorname{color}(\Gamma)$ and $i^{\left|\Gamma^{[0]}\right|+\left|\Gamma^{[1]}\right|}\left(\underline{g} \cos \theta_{W}\right)^{\left|P_{Z}^{[0]}\right|} \underline{e}^{\left|\Gamma^{[0]} \backslash P_{Z}^{[0]}\right|}$, respectively. Moreover, notice that due to the conservation of $W^{ \pm}$-particles each 3- or 4 -valent vertex has to consist of an even number of $W^{ \pm}$-particles. In particular, since the 4 -valent vertices are created by the Corolla polynomial in the same fashion than for the gluons we only need to consider the 3-valent Feynman rules, i.e. we have to sum over all possibilities to label edges in $\Gamma$ in such a way, that each 3-valent vertex consists of exactly two half-edges with label $W^{ \pm}$ and one unlabeled half-edge. Additionally the edge type has to be respected while gluing these 3 -valent vertices together. This is precisely fulfilled by the sets $\mathfrak{f} \in \mathfrak{F}_{2}(\Gamma)$. Furthermore, notice that the Feynman rules of the electroweak sector of the Standard Model allow each unlabeled edge of $\Gamma$ to be turned into either a $Z$-particle or an $A$-particle. This is precisely fulfilled by the sets $P_{Z} \in \mathscr{P}_{Z}(\mathfrak{f})$ for each set $\mathfrak{f} \in \mathfrak{F}_{2}(\Gamma)$. Moreover, the corresponding ghost edges have the same masses than their corresponding gauge bosons, meaning that each component $\mathcal{D}^{i}(\Gamma) I(\Gamma)$ gets the same factor $J\left(\Gamma ; m_{W}, m_{Z}\right)$ and thus the whole contribution created by the Corolla differential can be multiplied by the factor given in Equation (44), such that Equation (45) holds. Since no derivatives of $\mathcal{D}(\Gamma)$ act on the corresponding mass terms in the parametric representation of a scalar QFT, $I(\Gamma)$ can be simply multiplied by $J\left(\Gamma ; m_{W}, m_{Z}\right)$. Finally, the possibly different symmetry factors of $\Gamma_{\text {labeled }}$ compared to $\Gamma$ are compensated by the factor sym $(\Gamma) / \operatorname{sym}\left(\Gamma_{\text {labeled }}\right)$ and the redundant number of isomorphic graphs is divided out by iso $\left(\Gamma_{\text {labeled }}\right)$.

Lemma 5.13 (4]). Let the assumptions of Theorem 5.12 hold. Then we have

$$
\frac{\operatorname{sym}(\Gamma)}{\operatorname{sym}\left(\Gamma_{\text {labeled }}\right) \text { iso }\left(\Gamma_{\text {labeled }}\right)}=1, \quad \forall \Gamma .
$$

\footnotetext{
${ }^{19}$ Note that here the Corolla differential $\mathcal{D}(\Gamma)$ is used instead of $\mathcal{D}_{\mathrm{QCD}}(\Gamma)$, since the additional constants are moved into the definition of $J\left(\Gamma ; m_{W}, m_{Z}\right)$.
} 
Proof. Note that the symmetry factors of pure electroweak gauge boson Feynman graphs are similar to the symmetry factors of quantum electrodynamic Feynman graphs, since every vertex possesses exactly two orientable edges of the same type and one unoriented edge of another type. Since we have chosen to work in this paper with unoriented $W^{ \pm}$-labeled edges, the symmetry factors of pure electroweak gauge boson Feynman graphs are not all equal to 1, as in quantum electrodynamics for unoriented fermion edges [5]. Moreover, note that the labeling with $Z$-labels and $A$-labels does not change the symmetry-factors, i.e. the element $\mathfrak{f} \in \mathfrak{F}_{2}(\Gamma)$ has the same symmetry factor as all elements $P_{Z} \in \mathscr{P}_{Z}(\mathfrak{f})$. This is true because the symmetry factor can be defined as the number of ways half-edges of adjacent vertices can be interchanged without changing the graph. But since two edges of a vertex are of the same particle type, the remaining particle type is fixed and hence it does not matter if it is of $Z$-type or $A$-type, the only contribution comes from the unoriented two edges of the same particle type. Furthermore, we remark that as in quantum electrodynamics the sum of all possible orientations of unoriented edges (which is given by the factor iso $\left.\left(\Gamma_{\text {labeled }}\right)\right)$ times their symmetry factor sym $\left(\Gamma_{\text {labeled }}\right)$ is equal to 1 [5]. Moreover, when also ghost edges come into play, the symmetry factors may also change, but in the same way changes the number of isomorphic graphs with the same argument. Finally, in [3, Lemma 5.1] it is proven that the symmetry factors work out correct while passing from 3 -valent to 4 -valent vertices by shrinking internal edges.

Corollary $5.14([4])$. In particular, $J\left(\Gamma ; m_{W}, m_{Z}\right)$ simplifies to

$$
\begin{aligned}
J\left(\Gamma ; m_{W}, m_{Z}\right):= & \left(i^{\left|\Gamma^{[1]}\right|} \underline{g}_{s}{ }^{\left[\Gamma^{[0]} \mid\right.} \operatorname{color}(\Gamma)\right. \\
& +\sum_{\mathfrak{f} \in \mathfrak{F}_{2}(\Gamma) P_{Z} \in \mathscr{P}_{Z}(\mathfrak{f})} i^{\left|\Gamma^{[0]}\right|+\left|\Gamma^{[1]}\right|}\left(\underline{g} \cos \theta_{W}\right)\left|\underline{P}_{Z}^{[0]}\right| \underline{e}^{\left|\Gamma^{[0]} \backslash P_{Z}^{[0]}\right|} \\
& \left.\times e^{-\left(\sum_{e \in \mathfrak{f}}^{[1]} A_{e} m_{W}^{2}+\sum_{e \in P_{Z}^{[1]}} A_{e} m_{Z}^{2}\right)}\right) .
\end{aligned}
$$

Proof. Theorem 5.12 and Lemma 5.13 .

Example 5.15. Consider

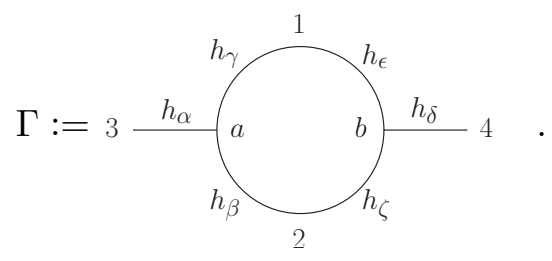


Then, we have (cf. Example 5.11):

$$
\begin{aligned}
J\left(3 h_{\alpha}{ }_{h_{\beta}}^{h_{\gamma}} \int_{h_{\zeta}}^{h_{\epsilon}} h_{\delta} m_{W}, m_{Z}\right)= & -\underline{g_{s}} f^{c_{3} c_{2} c_{1}} f^{c_{4} c_{2} c_{1}} \\
& +e^{-\left(\left(A_{3}+A_{2}+A_{4}\right) m_{W}^{2}\right)} \underline{e}^{2} \\
& +e^{-\left(\left(A_{3}+A_{2}+A_{4}\right) m_{W}^{2}+A_{1} m_{Z}^{2}\right)}\left(\underline{g} \cos \theta_{W}\right)^{2} \\
& +e^{-\left(\left(A_{3}+A_{1}+A_{4}\right) m_{W}^{2}\right)} \underline{e}^{2} \\
& +e^{-\left(\left(A_{3}+A_{1}+A_{4}\right) m_{W}^{2}+A_{2} m_{Z}^{2}\right)}\left(\underline{g} \cos \theta_{W}\right)^{2} \\
& +e^{-\left(\left(A_{1}+A_{2}\right) m_{W}^{2}\right)} \underline{e}^{2} \\
& +e^{-\left(\left(A_{1}+A_{2}\right) m_{W}^{2}+A_{4} m_{Z}^{2}\right)} \underline{e} \underline{g} \cos \theta_{W} \\
& +e^{-\left(\left(A_{1}+A_{2}\right) m_{W}^{2}+A_{3} m_{Z}^{2}\right)} \underline{e} \underline{g} \cos \theta_{W} \\
& \left.+e^{-\left(\left(A_{1}+A_{2}\right) m_{W}^{2}+\left(A_{3}+A_{4}\right) m_{Z}^{2}\right)}\left(\underline{g} \cos \theta_{W}\right)^{2}\right)
\end{aligned}
$$

\subsection{Inclusion of the scalar Bosons of the Electroweak Sector}

For the inclusion of the scalar bosons from the electroweak sector of the Standard Model we have to add four scalar particles, bringing new possible tensor structures in their Feynman rules with them. Concretely, we have to add the Higgs boson $h$ and the Goldstone bosons $\varphi^{ \pm}$and $\varphi_{Z} \cdot$

Outline. Again, the idea will be to define two nested sums over a 3-regular scalar QFT Feynman graph in the following way: The first sum creates all possible ways to attach scalar particle labels to edges of $\Gamma$ that are allowed to become a scalar edge (i.e. edges that will not become a ghost edge) and the second sum will create all possible Feynman graphs with 4-valent scalar vertices by shrinking suitable scalar labeled edges. Furthermore, we define a third sum which creates all particle labelings which are allowed by the Standard Model Feynman rules. The full details are explained in the proof after Theorem 5.20 .

Therefore, we define:

Definition 5.16. Let $\Gamma$ be a 3-regular scalar QFT Feynman graph. Then:

1. Again, as in Definition [5.10, let $\wp\left(\Gamma^{[1]}\right)$ be the power set of all external edges and internal edges of $\Gamma$ with all adjacent vertices added. In particular, we are interested in the set $\wp\left(\Gamma^{[1]} \backslash\left(\bigcup_{k=1}^{i} C_{i}^{[1]}\right)\right)$, the power set of all edges of $\Gamma$ that will not be turned into ghost edges and are free to possibly become a Higgs or Goldstone edge. We denote the elements of $\wp\left(\Gamma^{[1]} \backslash\left(\bigcup_{k=1}^{i} C_{i}^{[1]}\right)\right)$ by $P_{\mathrm{H} / \mathrm{G}}$.

2. Let $\wp_{(4)}\left(P_{\mathrm{H} / \mathrm{G}}\right)$ be the power set of all internal edges of $\Gamma$ in the set $P_{\mathrm{H} / \mathrm{G}}$ having 2 or 4 neighbors, that do not share a vertex with an edge which will be turned into a ghost edge (also edges that will be turned into fermions edges are not allowed either, if included). 
Also adjacent edges are not allowed to be in the same set $P_{(4)} \in \wp_{(4)}\left(P_{\mathrm{H} / \mathrm{G}}\right)$. The set of sets $\wp_{(4)}\left(P_{\mathrm{H} / \mathrm{G}}\right)$ consists therefore of all edges in the set $P_{\mathrm{H} / \mathrm{G}}$ that are free to create a valid 4-valent vertex (either 2-scalar-2-gauge boson or 4-scalar boson vertices). Again, add all adjacent vertices in each edge set.

3. Let $e \in P_{(4)}\left(P_{\mathrm{H} / \mathrm{G}}\right)$. Then we define the set of adjacent half-edges to $e$ as $H_{(4)}(e):=$ $\left\{h_{1}, h_{2}, h_{3}, h_{4}\right\}$. In particular, we are interested in the set $H_{(2)}(e) \subset H_{(4)}(e)$, defined as

$$
H_{(2)}(e):= \begin{cases}\left\{h_{1}, h_{2}\right\} & \text { if } h_{1}, h_{2} \notin P_{\mathrm{H} / \mathrm{G}} \text { and } h_{3}, h_{4} \in P_{\mathrm{H} / \mathrm{G}} \\ \emptyset & \text { if } h_{1}, h_{2}, h_{3}, h_{4} \in P_{\mathrm{H} / \mathrm{G}}\end{cases}
$$

for some arbitrary numbering $1, \ldots, 4$, since in the case of a 2 -scalar-2-gauge boson vertex we have to add a metric tensor with indices depending on the gauge boson edges (where the product below in the modified Corolla polynomial in Definition 5.19 over the empty set is defined to be 0 ). For the 4 -scalar boson vertex we just receive a coupling constant, cf. part 5. of this definition.

4. Let $\mathscr{L}\left(\Gamma, P_{\mathrm{H} / \mathrm{G}}, P_{(4)}\right)$ denote the set of all possible allowed particle type labelings of the graph $\Gamma$, with scalar particle edges $P_{\mathrm{H} / \mathrm{G}}$ and 4-valent scalar vertices $P_{(4)}$, compatible with the Standard Model Feynman rules (cf. [4, Appendix A] and [8]), such that 20

$$
L(e) \in\left\{W^{ \pm}, Z, A, h, \varphi^{ \pm}, \varphi_{Z}\right\}, \quad e \in \Gamma^{[1]} .
$$

In the previous Subsection 5.2 this was created by the sets of sets $\mathfrak{F}_{2}(\Gamma)$ and $\mathscr{P}_{Z}(\mathfrak{f})$ for the special cases $P_{\mathrm{H} / \mathrm{G}}=P_{(4)}=\emptyset$, defined in Definition 5.10,

5. Let coupling $\left(P_{\mathrm{H} / \mathrm{G}}, P_{(4)}, L\right)$ be the product over all factors of $\pm i$ and all coupling constants given by the Feynman rules for $\Gamma$ with labeling $L$ (cf. [4, Appendix A] and [8]). The coupling constants include all 3 -valent vertices and 4-scalar boson vertices (the 4-gauge boson vertex coupling constants are created from the corresponding 3-valent ones by the Corolla polynomial via graph homology as usual).

We start with examples for 1., 2. and 3. from Definition 5.16.

Example 5.17. Again, we consider the one-loop graph

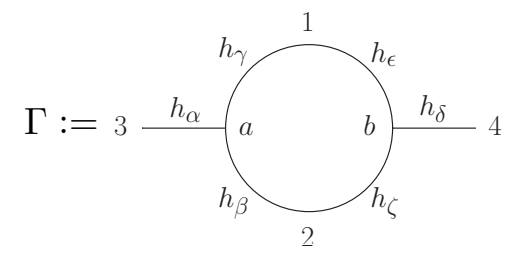

and

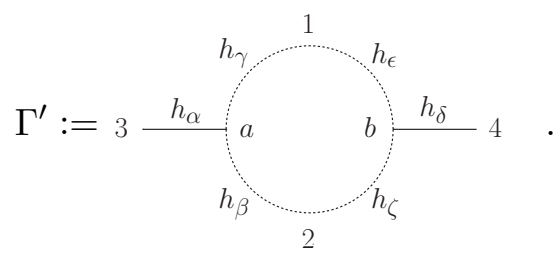

\footnotetext{
${ }^{20}$ We denote here the gauge bosons as well as their corresponding ghosts, by $\left\{W^{ \pm}, Z, A\right\}$ since in the following we are only interested in their pairwise coincident masses.
} 
Then, we have:

$$
\begin{aligned}
& \wp\left(\Gamma^{[1]}\right)=\{\underbrace{\emptyset}_{P_{\mathrm{H} / \mathrm{G}}^{(1)}(\Gamma)}, \underbrace{\{a, b ; 1\}}_{P_{\mathrm{H} / \mathrm{G}}^{(2)}(\Gamma)}, \underbrace{\{a, b ; 2\}}_{P_{\mathrm{H} / \mathrm{G}}^{(3)}(\Gamma)}, \underbrace{\{a ; 3\}}_{P_{\mathrm{H} / \mathrm{G}}^{(4)}(\Gamma)}, \underbrace{\{b ; 4\}}_{P_{\mathrm{H} / \mathrm{G}}^{(5)}(\Gamma)}, \underbrace{\{a, b ; 1,2\}}_{P_{\mathrm{H} / \mathrm{G}}^{(6)}(\Gamma)}, \underbrace{\{a, b ; 1,3\}}_{P_{\mathrm{H} / \mathrm{G}}^{(7)}(\Gamma)}, \\
& \underbrace{\{a, b ; 1,4\}}_{P_{\mathrm{H} / \mathrm{G}}^{(8)}(\Gamma)}, \underbrace{\{a, b ; 2,3\}}_{P_{\mathrm{H} / \mathrm{G}}^{(9)}(\Gamma)}, \underbrace{\{a, b ; 2,4\}}_{P_{\mathrm{H} / \mathrm{G}}^{(10)}(\Gamma)}, \underbrace{\{a, b ; 3,4\}}_{P_{\mathrm{H} / \mathrm{G}}^{(11)}(\Gamma)}, \underbrace{\{a, b ; 1,2,3\}}_{P_{\mathrm{H} / \mathrm{G}}^{(12)}(\Gamma)}, \\
&\underbrace{\{a, b ; 1,2,4\}}_{P_{\mathrm{H} / \mathrm{G}}^{(13)}(\Gamma)}, \underbrace{\{a, b ; 1,3,4\}}_{P_{\mathrm{H} / \mathrm{G}}^{(14)}(\Gamma)}, \underbrace{\{a, b ; 2,3,4\}}_{P_{\mathrm{H} / \mathrm{G}}^{(15)}(\Gamma)}, \underbrace{\{a, b ; 1,2,3,4\}}_{P_{\mathrm{H} / \mathrm{G}}^{(16)}(\Gamma)}\} \\
& \wp\left(\Gamma^{\prime[1]}\right)=\wp\left(\Gamma^{[1]} \backslash C_{1}^{(1])}\right)=\{\underbrace{\emptyset}_{P_{\mathrm{H} / \mathrm{G}}^{(1) !}\left(\Gamma^{\prime}\right)}, \underbrace{\{a ; 3\}}_{P_{\mathrm{H} / \mathrm{G}}^{(2) \prime}\left(\Gamma^{\prime}\right)}, \underbrace{\{b ; 4\}}_{P_{\mathrm{H} / \mathrm{G}}^{(3) \prime}\left(\Gamma^{\prime}\right)}, \underbrace{\{a, b ; 3,4\}}_{P_{\mathrm{H} / \mathrm{G}}^{(4) \prime}\left(\Gamma^{\prime}\right)}\}
\end{aligned}
$$

and

$$
\begin{aligned}
& \wp_{(4)}\left(P_{\mathrm{H} / \mathrm{G}}^{(l)}\right)=\{\underbrace{\emptyset}_{P_{(4)}^{(1)}\left(P_{\mathrm{H} / \mathrm{G}}^{(l)}\right)}, \quad 1 \leq l \leq 13 \\
& \wp_{(4)}\left(P_{\mathrm{H} / \mathrm{G}}^{(14)}\right)=\{\underbrace{\emptyset}_{P_{(4)}^{(1)}\left(P_{\mathrm{H} / \mathrm{G}}^{(14)}\right)}, \underbrace{\{a, b ; 1\}}_{P_{(4)}^{(2)}\left(P_{\mathrm{H} / \mathrm{G}}^{(14)}\right)}\} \\
& \wp_{(4)}\left(P_{\mathrm{H} / \mathrm{G}}^{(15)}\right)=\{\underbrace{\emptyset}_{P_{(4)}^{(1)}\left(P_{\mathrm{H} / \mathrm{G}}^{(15)}\right)}, \underbrace{\{a, b ; 2\}}_{P_{(4)}^{(2)}\left(P_{\mathrm{H} / \mathrm{G}}^{(15)}\right)}\} \\
& \wp_{(4)}\left(P_{\mathrm{H} / \mathrm{G}}^{(16)}\right)=\{\underbrace{\emptyset}_{P_{(4)}^{(1)}\left(P_{\mathrm{H} / \mathrm{G}}^{(16)}\right)}, \underbrace{\{a, b ; 1\}}_{P_{(4)}^{(2)}\left(P_{\mathrm{H} / \mathrm{G}}^{(16)}\right)} \underbrace{\{a, b ; 2\}}_{P_{(4)}^{(3)}\left(P_{\mathrm{H} / \mathrm{G}}^{(16)}\right)}\} \\
& \wp_{(4)}\left(P_{\mathrm{H} / \mathrm{G}}^{(l) \prime}\right)=\{\underbrace{\emptyset}_{P_{(4)}^{(1)}\left(P_{\mathrm{H} / \mathrm{G}}^{(l) \prime}\right)}\}, \quad 1 \leq l \leq 4
\end{aligned}
$$

And for our sets $H_{(2)}(e), e \in P_{(4)}\left(P_{\mathrm{H} / \mathrm{G}}\right)$, we have (we just list the non-empty sets):

$$
\begin{array}{ll}
1 \in P_{(4)}^{(1)}\left(P_{\mathrm{H} / \mathrm{G}}^{(6)}\right): & H_{(2)}(1)=\left\{h_{\alpha}, h_{\delta}\right\} \\
2 \in P_{(4)}^{(1)}\left(P_{\mathrm{H} / \mathrm{G}}^{(6)}\right): & H_{(2)}(2)=\left\{h_{\alpha}, h_{\delta}\right\}
\end{array}
$$




$$
\begin{array}{ll}
1 \in P_{(4)}^{(1)}\left(P_{\mathrm{H} / \mathrm{G}}^{(14)}\right): & H_{(2)}(1)=\left\{h_{\beta}, h_{\zeta}\right\} \\
2 \in P_{(4)}^{(1)}\left(P_{\mathrm{H} / \mathrm{G}}^{(15)}\right): & H_{(2)}(2)=\left\{h_{\gamma}, h_{\epsilon}\right\}
\end{array}
$$

These create, after applying the Corolla differential for the electroweak sector of the Standard Model (cf. Definition 5.19 and Theorem 5.20) to the scalar parametric integrand $I(\Gamma)$, the tensor structure contributions of the following gauge theory graphs (which is indicated by the arrow "œ"):

$$
P_{(4)}^{(1)}\left(P_{\mathrm{H} / \mathrm{G}}^{(1)}\right)
$$




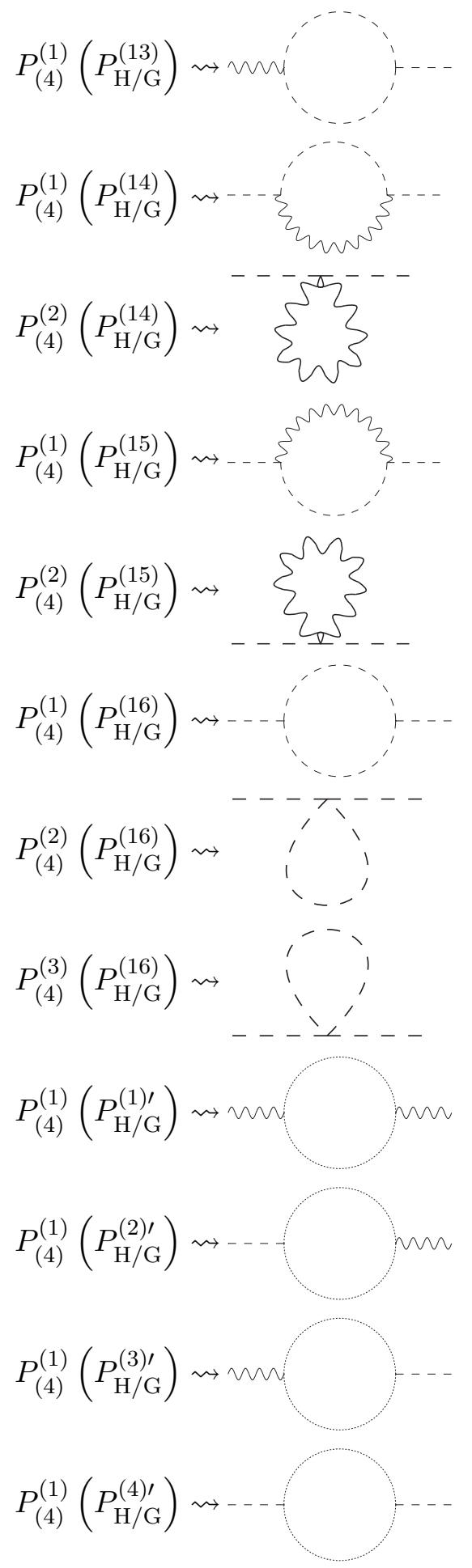

(54m)

Furthermore, we have the following examples for 4. and 5. of Definition 5.16 . 
Example 5.18. We have:

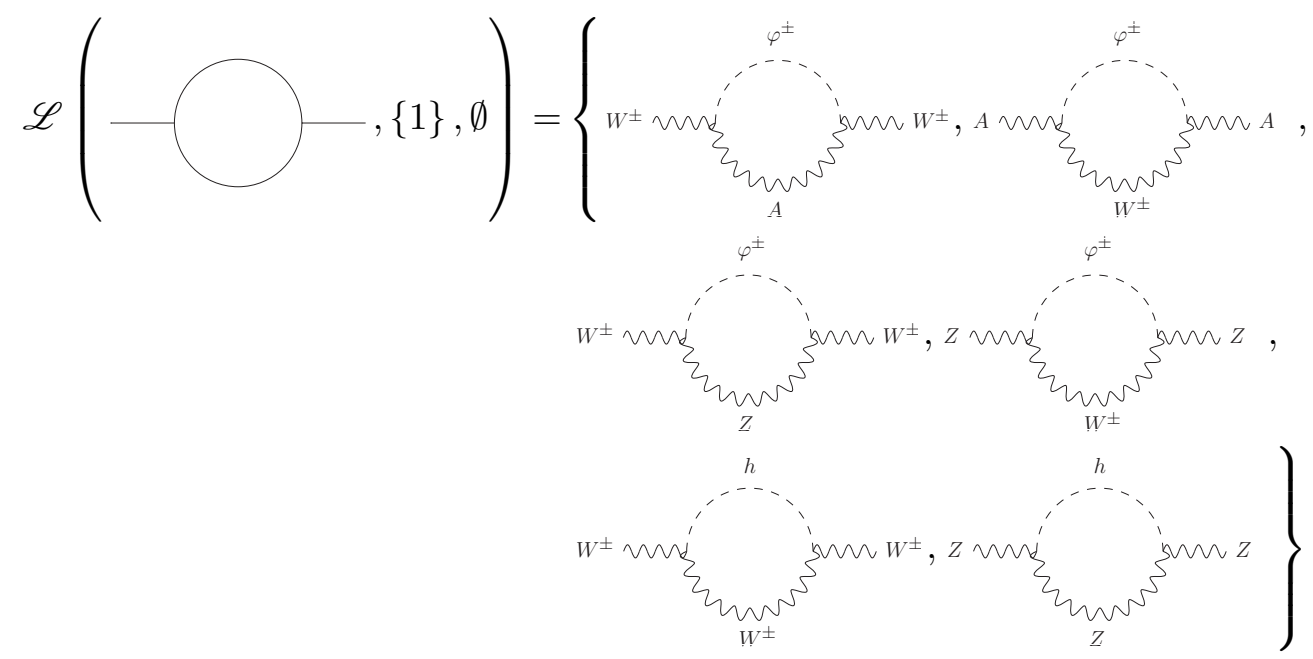

and:

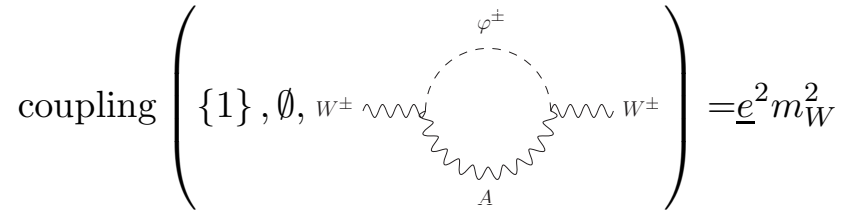

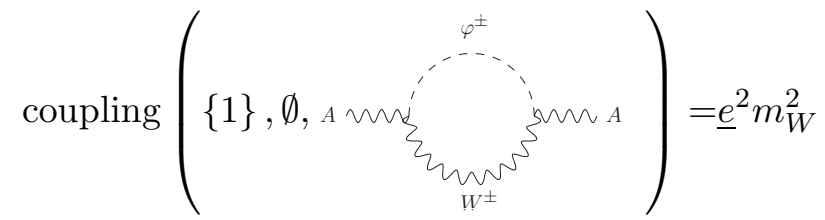

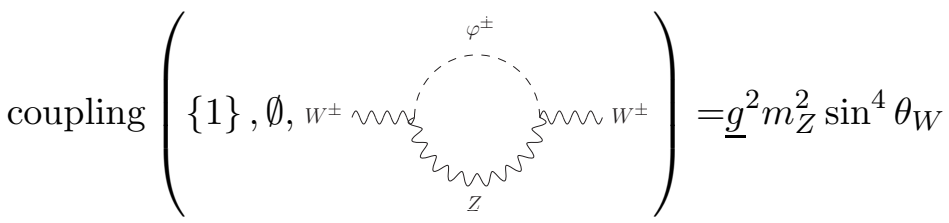

$$
\begin{aligned}
& \text { coupling }\left(\{1\}, \emptyset, z m \underline{g}_{W^{ \pm}} \underline{g}^{2} m_{Z}^{2} \sin ^{4} \theta_{W}\right.
\end{aligned}
$$

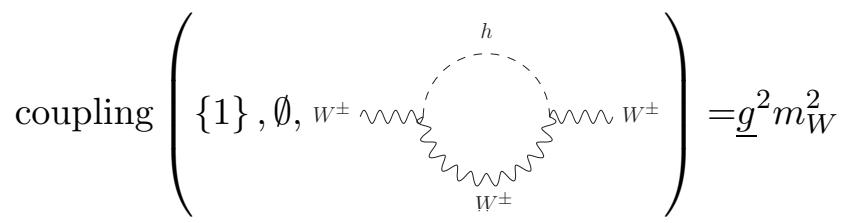

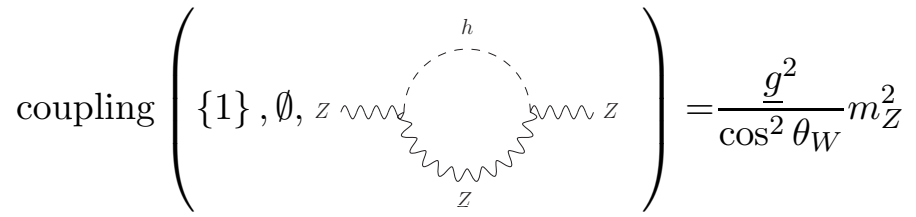

Then we can define the Corolla polynomial for the gauge bosons and the scalar particles of the electroweak sector of the Standard Model as follows:

Definition 5.19 (Corolla polynomial for the electroweak sector of the Standard Model (omitting fermions)). Let $\Gamma$ be a 3-regular scalar QFT Feynman graph. Then we define the various 
summands of the Corolla polynomial for the electroweak sector of the Standard Model (omitting fermions) by

$$
\begin{aligned}
& \mathcal{C}_{\mathrm{EW}}^{0}(\Gamma):=\sum_{P_{\mathrm{H} / \mathrm{G}} \in \wp\left(\Gamma^{[1]}\right)} \sum_{P_{(4)} \in \wp_{\wp}(4)} \sum_{\left(P_{\mathrm{H} / \mathrm{G}}\right)} \sum_{L \in \mathscr{L}\left(\Gamma, P_{\mathrm{H} / \mathrm{G}}, P_{(4)}\right)} \\
& {\left[\left(\frac{\operatorname{sym}(\Gamma)}{\operatorname{sym}(L) \text { iso }(L)}\right) \text { coupling }\left(P_{\mathrm{H} / \mathrm{G}}, P_{(4)}, L\right)\right.} \\
& e^{\left(\sum_{e \in P_{(4)}^{[1]}} A_{e} \xi_{e}^{\prime 2}-\sum_{e \in \Gamma^{[1]} \backslash P_{(4)}^{[1]}} A_{e} m_{L(e)}^{2}\right)}
\end{aligned}
$$

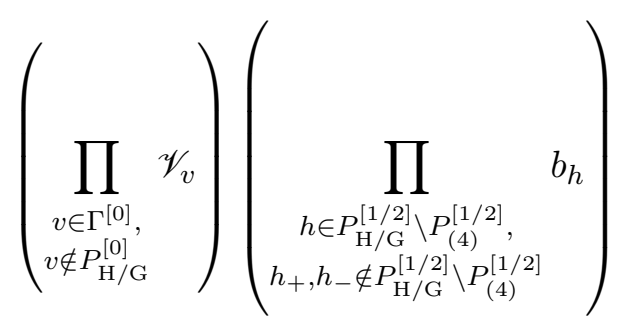

$$
\begin{aligned}
& \left.\left(\prod_{\substack{h_{+}, h_{-} \in P_{1 / \mathrm{G}}^{[1 / 2]} \backslash P_{(4)}^{[1 / 2]}, h \notin P_{\mathrm{H} / \mathrm{G}}^{[1 / 2]} \backslash P_{(4)}^{[1 / 2]}}}\left(a_{h_{+}}+a_{h_{-}}\right)\right)\left(\prod_{\substack{e \in P_{(4)}^{[1]}, h_{1}, h_{2} \in H_{(2)}(e)}} b_{h_{1}} b_{h_{2}}\right)\right], \\
& \mathcal{C}_{\mathrm{EW}}^{i}(\Gamma):=\sum_{\substack{C_{1}, C_{2}, \ldots, C_{i} \in \mathscr{C}_{\Gamma}, C_{j} \text { pairwise disjoint }}} \sum_{P_{\mathrm{H} / \mathrm{G}} \in \wp(} \sum_{\left(\Gamma^{[1]} \backslash \bigcup_{k=1}^{i}, C_{k}^{[1]}\right)} \sum_{P_{(4)} \in \wp_{(4)}\left(P_{\mathrm{H} / \mathrm{G}}\right)} \sum_{L \in \mathscr{L}\left(\Gamma, P_{\mathrm{H} / \mathrm{G}}, P_{(4)}\right)} \\
& {\left[\left(\frac{\operatorname{sym}(\Gamma)}{\operatorname{sym}(L) \operatorname{iso}(L)}\right) \operatorname{coupling}\left(P_{\mathrm{H} / \mathrm{G}}, P_{(4)}, L\right)\right.} \\
& e^{\left(\sum_{e \in P_{(4)}^{[1]}} A_{e} \xi_{e}^{\prime 2}-\sum_{e \in \Gamma^{[1]} \backslash P_{(4)}^{[1]}} A_{e} m_{L(e)}^{2}\right)}
\end{aligned}
$$

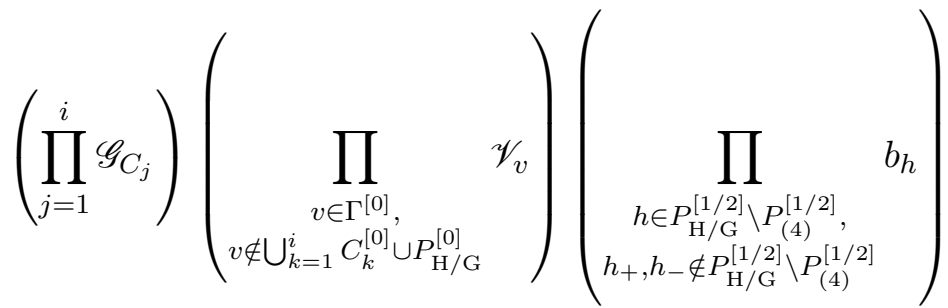

$$
\begin{aligned}
& \left.\left(\prod_{\substack{h_{+}, h_{-} \in P_{\mathrm{H} / \mathrm{G}}^{[1 / 2]} \backslash P_{(4)}^{[1 / 2]}, h \notin P_{\mathrm{H} / \mathrm{G}}^{[1 / 2]} \backslash P_{(4)}^{[1 / 2]}}}\left(a_{h_{+}}+a_{h_{-}}\right)\right)\left(\prod_{\substack{e \in P_{(4)}^{[1],},(e) \\
h_{1}, h_{2} \in H_{(2)}(e)}} b_{h_{1}} b_{h_{2}}\right)\right]
\end{aligned}
$$

and the Corolla polynomial by

$$
\mathcal{C}_{\mathrm{EW}}(\Gamma):=\sum_{i=0}^{\infty}(-1)^{i} \mathcal{C}_{\mathrm{EW}}^{i}(\Gamma)
$$


Theorem 5.20 (4] ). Let $\Gamma$ be a connected 21 3-regular scalar QFT Feynman graph. Then, the parametric integrand of all Feynman graphs from quantum chromodynamics and the electroweak sector of the Standard Model which are related to $\Gamma$ via graph and cycle homology including all possible particle type labelings allowed by the Standard Model Feynman rules (omitting fermions) $\tilde{I}_{\mathcal{F}}(\Gamma)$ can be obtained via acting with the sum of the two Corolla differentials for quantum chromodynamics and the electroweak sector of the Standard Model $\left(\mathcal{D}_{\mathrm{QCD}}(\Gamma)+\mathcal{D}_{\mathrm{EW}}(\Gamma)\right)$ on the corresponding parametric integrand $I(\Gamma)$ of $\mathrm{I} 22$,

$$
\tilde{I}_{\mathcal{F}}(\Gamma)=\left(\mathcal{D}_{\mathrm{QCD}}(\Gamma)+\mathcal{D}_{\mathrm{EW}}(\Gamma)\right) I(\Gamma)
$$

Proof. First note that the scalar particles $h, \varphi^{ \pm}$and $\varphi_{Z}$ only couple to gauge bosons of the electroweak sector of the Standard Model so that we are allowed to treat it separately from quantum chromodynamics. The contribution for quantum chromodynamics via the action from $\mathcal{D}_{\mathrm{QCD}}(\Gamma)$ on $I(\Gamma)$ was already proven in Theorem 5.7. For the contribution of the electroweak sector of the Standard Model notice that for a given graph $\Gamma$ every edge that is not going to be turned into a ghost edge is allowed to become a scalar edge (possibly with a restriction on the edge labeling). This is precisely created by the power set of all edges of the graph $\Gamma$ without the edges that will be turned into ghost edges, i.e. $\wp\left(\Gamma^{[1]} \backslash \bigcup_{k=1}^{i} C_{k}^{[1]}\right)$ for the contribution with $i$ ghost loops. Moreover, the creation of 4-valent scalar vertices (2-scalar-2-gauge boson or 4-scalar boson vertices) can be constructed via shrinking suitable internal scalar labeled edges between two 3 -valent vertices. Concrete, the requirements are having 2 or 4 scalar edge neighbors, i.e. edges in the set $P_{\mathrm{H} / \mathrm{G}}$. Furthermore, they are not allowed to share a vertex with an edge which will be turned into a ghost edge (again, we remark that also edges which will be turned into fermion edges are not allowed either, if included). And finally, also adjacent edges are not allowed to shrink simultaneously. Therefore, $\wp_{(4)}\left(P_{\mathrm{H} / \mathrm{G}}\right)$ consists of all sets of edges that are allowed to shrink in the set $P_{\mathrm{H} / \mathrm{G}}$ in order to produce valid 4 -valent scalar vertices. Furthermore, the Feynman rules for the 3-valent 1-scalar-2-gauge boson and 2-scalar-1-gauge boson vertices are created by the additional products of the Corolla polynomial for the electroweak sector of the Standard Model (cf. Definition 5.19 compared to Definition [5.1). Moreover, since the original Corolla polynomial acts only on the part(s) of $\Gamma$ which will be turned into pure gauge boson vertices or their corresponding ghosts, the 4-valent 4-gauge boson vertices are created just like with the standard Corolla differential and can be received as the corresponding residues in the shrinked edge Schwinger parameters, cf. Theorem 5.7. Additionally, the right symmetry factors are obtained by the multiplication with the fraction $\operatorname{sym}(\Gamma) / \operatorname{sym}(L)$ and possible redundancies are divided out by the factor iso $(L)$. The corresponding particle masses are included via the exponential term which also removes the Schwinger parameters for shrinked scalar labeled edges. Finally all corresponding coupling constants for the labeling $L \in \mathscr{L}\left(\Gamma, P_{\mathrm{H} / \mathrm{G}}, P_{(4)}\right)$ are given in coupling $\left(P_{\mathrm{H} / \mathrm{G}}, P_{(4)}, L\right)$.

\section{Conclusion}

The aim of this paper was to review the generalization of the Corolla polynomial defined in 1, 2, 3 to the bosons of the electroweak sector of the Standard Model, which was first worked out in [4]. Therefore, all the relevant graph theoretic notions (cf. Section 3), the parametric representation of scalar quantum field theories (cf. Section 4) and the Corolla polynomial and differential for pure Yang-Mills theory (cf. Subsection 5.1) were reviewed. The inclusion of the bosons of the electroweak sector of the Standard Model was made possible in two steps. First by working out

\footnotetext{
${ }^{21}$ Again, we emphasize that we really mean 1-connected graphs, not only 2-connected (as they are called in mathematics) or 1 PI (as they are called in physics) graphs.

${ }^{22}$ Recall $\mathcal{D}_{\mathrm{QCD}}(\Gamma):=i^{\mid \Gamma^{[1]}}\left|\underline{g}_{s}\right| \Gamma^{[0]} \mid \operatorname{color}(\Gamma) \mathcal{D}(\Gamma)$ from Definition 5.1 and Definition 5.5
} 
the combinatorics of labeling a 3-regular scalar QFT Feynman graph with labels of the gauge bosons of the electroweak sector of the Standard Model (cf. Subsection 5.2). Then secondly by working out the additional tensor structures arising from the inclusion of the Feynman rules for the scalar particles of the electroweak sector of the Standard Model (cf. Subsection 5.3). We showed that at least for the gauge bosons of the electroweak sector of the Standard Model the symmetry factors work out correct (cf. Corollary [5.14). Furthermore, a relatively compact notation was given for the inclusion of the scalar particles of the Standard Model, compared to the standard Standard Model Feynman rules (cf. Definition 5.19, Definition 5.5, Theorem 5.20, [4, Appendix A] and [8]).

As projects for future work, several topics related to the Corolla polynomial are of particular interest: First of all, although it is in principle clear [2], the combinatorics for the inclusion of fermions to the Corolla polynomial for the electroweak sector of the Standard Model should be explicitly worked out. Secondly, as we believe that this approach is useful for computer calculations - since derivations can be done with much less computational afford than integrations it would be useful to bring this approach on a computer. And thirdly, since it is believed that the Corolla polynomial can also be generalized to the case of quantum gravity [2, it would be interesting to see what we could learn from this approach and its underlying combinatorics.

\section{Acknowledgments}

It is my pleasure to thank Prof. Dr. Dirk Kreimer, Dr. Christian Bogner and the rest of the group for the welcoming atmosphere, their support and illuminating and helpful discussions!

\section{References}

[1] D. Kreimer and K. Yeats: Properties of the Corolla Polynomial of a 3-regular Graph. Electr. J. Comb., pages 41-41, 2013. https://arxiv.org/abs/1207.5460v1.

[2] D. Kreimer, M. Sars, and W. D. van Suijlekom: Quantization of gauge fields, graph polynomials and graph homology. Annals Phys., 336:180-222, 2013. https://arxiv.org/abs/1208.6477v4.

[3] M. Sars: Parametric Representation of Feynman Amplitudes in Gauge Theories. $\quad \mathrm{PhD}$ thesis, Humboldt-Universität $\mathrm{zu}$ Berlin, January 2015. https://www2.mathematik.hu-berlin.de/ kreimer/wp-content/uploads/SarsThesisNeu.pdf.

[4] D. Prinz: The Corolla Polynomial for spontaneously broken Gauge Theories. Master thesis, September 2015. https://www2.mathematik.hu-berlin.de/ kreimer/wp-content/uploads/prinz.pdf.

[5] D. Kreimer: Dirk Kreimer's lecture series (QFT I, QFT II, Hopf Algebras and the renormalization group, Dyson-Schwinger equations and quantization of gauge theories). https://www2.mathematik.hu-berlin.de/ kreimer/teaching/, Scripts for the latter two by Lutz Klaczynski can be found here https://www2.mathematik.hu-berlin.de/ klacz/SkriptRGE.pdf and here https://www2.mathematik.hu-berlin.de/ klacz/DSE.pdf.

[6] M. E. Peskin and D. V. Schroeder: An Introduction to Quantum Field Theory. Advanced book classics. Addison-Wesley Publishing Company, 1995, ISBN 9780201503975. https://books.google.de/books?id=i35LALNOGosC

[7] C. Itzykson and J. B. Zuber: Quantum Field Theory. Dover Books on Physics. Dover Publications, 2012, ISBN 9780486134697. https://books.google.de/books?id=CxYCMNrUnTEC 
[8] J. C. Romão and J. P. Silva: A resource for signs and Feynman diagrams of the Standard Model. arXiv, arXiv:1209.6213v2, October 2012. https://arxiv.org/abs/1209.6213v2.

[9] W. D. van Suijlekom: Renormalization of Gauge Fields: A Hopf Algebra Approach. Commun. Math. Phys., 276:773-798, April 2007. https://arxiv.org/abs/hep-th/0610137v1. Digital Object Identifier (DOI) 10.1007/s00220-007-0353-9.

[10] R. Diestel: Graph Theory, 4th Edition, volume 173 of Graduate texts in mathematics. Springer, 2012, ISBN 978-3-642-14278-9. https://diestel-graph-theory.com/.

[11] F. Brown and D. Kreimer: Angles, Scales and Parametric Renormalization. Letters in Mathematical Physics, 103(9):933-1007, 2013, ISSN 0377-9017. https://arxiv.org/abs/1112.1180v1. 